\title{
A Game Theoretic Model for the Formation of Navigable Small-World Networks - the Tradeoff between Distance and Reciprocity
}

ZHI YANG, Peking University

WEI CHEN, Microsoft Research

Kleinberg proposed a family of small-world networks to explain the navigability of large-scale real-world social networks. However, the underlying mechanism that drives real networks to be navigable is not yet well understood. In this paper, we present a game theoretic model for the formation of navigable small world networks. We model the network formation as a game called the Distance-Reciprocity Balanced (DRB) game in which people seek for both high reciprocity and long-distance relationships. We show that the game has only two Nash equilibria: One is the navigable small-world network, and the other is the random network in which each node connects with each other node with equal probability, and any other network state can reach the navigable small world via a sequence of best-response moves of nodes. We further show that the navigable small world equilibrium is very stable - (a) no collusion of any size would benefit from deviating from it; and (b) after an arbitrary deviations of a large random set of nodes, the network would return to the navigable small world as soon as every node takes one best-response step. In contrast, for the random network, a small group collusion or random perturbations is guaranteed to bring the network out of the random-network equilibrium and move to the navigable network as soon as every node takes one best-response step. Moreover, we show that navigable small world equilibrium has much better social welfare than the random network, and provide the price-of-anarchy and price-of-stability results of the game. Our empirical evaluation further demonstrates that the system always converges to the navigable network even when limited or no information about other players' strategies is available, and the DRB game simulated on real-world networks leads to navigability characteristic that is very close to that of the real networks, even though the real-world networks have non-uniform population distributions different from the Kleinberg's small-world model. Our theoretical and empirical analyses provide important new insight on the connection between distance, reciprocity and navigability in social networks.

Categories and Subject Descriptors: G.2.2 [Discrete Mathematics]: Graph Theory-Network problems

Additional Key Words and Phrases: Small-world network, game theory, navigability, reciprocity

\section{INTRODUCTION}

In 1967, Milgram published his work on the now famous small-world experiment [Milgram 1967]: he asked test subjects to forward a letter to their friends in order for the letter to reach a person not known to the initiator of the letter. He found that on average it took only six hops to connect two people in U.S., which is often attributed as the source of the popular term six-degree of separation. This seminal work inspired numerous studies on the small-world phenomenon and small-world models, which last till the present day of information age.

In [Watts and Strogatz 1998] Watts and Strogatz investigated a number of real-world networks such as film actor networks and power grids, and showed that many networks have both low diameter and high clustering (meaning two neighbors of a node are likely to

A preliminary version of this work appeared in Proceedings of the 24th International World Wide Web Conference (WWW 2015). The current version contains a number of new results comparing to the preliminary version, such as heterogeneous utility functions, analytical results on the best-response dynamics, non-existence of non-uniform equilibria, price of anarchy and price of stability, and empirical evaluation on real datasets.

The work was partly done when the first author was a visiting researcher at Microsoft Research Asia. This work was supported by the National Basic Research Program of China (Grant No. 2014CB340400).

Author's addresses: Zhi Yang, Computer Science Department, Peking University, Beijing, China; email:yangzhi@pku.edu.cn. Wei Chen, Microsoft Research, Beijing, China; email:weic@microsoft.com.

YYYY 0000-0000/YYYY/01-ARTA $\$ 15.00$

DOI: http://dx.doi.org/10.1145/0000000.0000000 


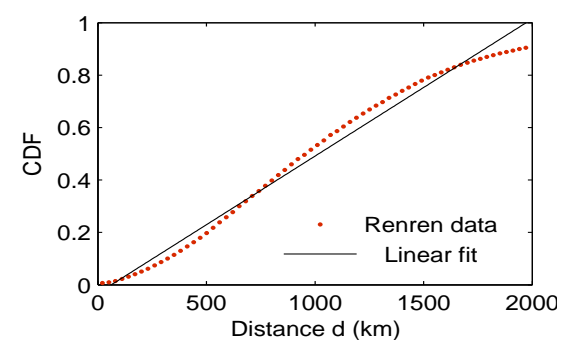

Fig. 1. The fraction of nodes within distance $d$ in Renren.

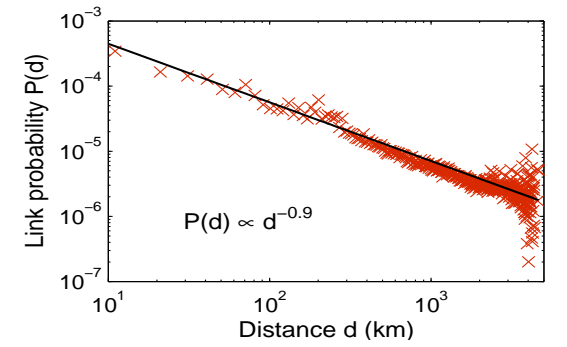

Fig. 2. Friendship probability vs. distance in Renren.

be neighbors of each other), which is different from randomly wired networks. They thus proposed a small-world model in which nodes are first placed on a ring or a grid with local connections, and then some connections are randomly rewired to connect to longrange contacts in the network. The local and long-range connections can also be viewed as strong ties and weak ties respectively in social relationships originally proposed by Granovetter [Granovetter 1973; Granovetter 1974].

Kleinberg notices an important discrepancy between the small-world model of Watts and Strogatz and the original Milgram experiment: the latter shows not only that the average distance between nodes in the network are small, but also that a decentralized routing algorithm using only local information can construct short paths. Here, we call a routing algorithm decentralized in that given a source node $u$ and a destination node $v$, the algorithm attempts to come up with a path $u=x_{0}, x_{1}, x_{2}, \ldots, x_{m}=v$, only using the acquaintance relationships of these $m$ intermediate nodes $x_{0}, x_{1}, x_{2}, \ldots, x_{m-1}$. By contrast, a centralised algorithm (e.g., Dijkstra's algorithm) requires the nodes to know full network (i.e., the acquaintance relationships among all people in the world) to find an optimal route, but obviously they cannot know this in real networks.

To address this issue, Kleinberg adjusted the Watts-Strogatz model so that the longrange connections are selected not uniformly at random among all nodes but inversely proportional to a power of the grid distance between the two end points of the connection [Kleinberg 2002]. More specifically, Kleinberg modeled a social network as composed of $n^{k}$ nodes on a $k$-dimensional grid, with each node having local contacts to other nodes in its immediate geographic neighborhood. Each node $u$ also establishes a number of long-range contacts, and a long-range link from $u$ to $v$ is established with probability proportional to $d_{M}(u, v)^{-r}$, where $d_{M}(u, v)$ is the grid distance between $u$ and $v$, and $r \geq 0$ is the model parameter indicating how likely nodes prefer to connect to remote nodes, which we call connection preference in the paper. The Watts-Strogatz model corresponds to the case of $r=0$, and as $r$ increases, nodes are more likely to connect to other nodes in their vicinity. Kleinberg modeled Milgram's experiment as decentralized greedy routing in such networks, in which each node only forwards messages to one of its neighbors with coordinate closest to the target node. He showed that when $r=k$, greedy routing can be done efficiently in $O\left(\log ^{2} n\right)$ time in expectation, but for any $r \neq k$, it requires $\Omega\left(n^{c}\right)$ time for some constant $c$ depending on $r$, exponentially worse than the case of $r=k$. Therefore, the small world at the critical value of $r=k$ is meant to model the real-world navigable network validated by Milgram and others' experiments, and we call it the navigable small-world network.

After Kleinberg's theoretical analysis, a number of empirical studies have been conducted to verify if real networks indeed have connection preferences close to the critical value that allows efficient greedy routing [Liben-Nowell et al. 2005; Adamic and Adar 2005; Cho et al. 2011; Goldenberg and Levy 2009; Schaller and Latank 1995]. Since real population is not evenly distributed geographically as in the Kleinberg's model, Liben-Nowell et al. [Liben-Nowell et al. 2005] proposed to use the fractional dimension D, defined as 
the best value to fit $\left|\left\{w: d_{M}(u, w) \leq d_{M}(u, v)\right\}\right|=c \cdot d_{M}(u, v)^{D}$, averaged over all $u$ and $v$. They showed that when the connection preference $r=D$, the network is navigable. They then studied a network of 495,836 LiveJournal users in the continental United States who list their hometowns, and find that $D \approx 0.8$ while $r=1.2$, reasonably close to $D$. We apply the same approach to a ten million node Renren network [Jiang et al. 2010; Yang et al. 2011], one of the largest online social networks in China. We map the hometown listed in users' profiles to (longitude, latitude) coordinates. The resolution of our geographic data is limited to the level of towns and cities and thus we cannot get the exact distance of nodes within $10 \mathrm{~km}$. We found that $D \approx 1$ (Figure 1 ) and $r \approx 0.9$ (Figure 2) in the Renren network. Other studies [Adamic and Adar 2005; Cho et al. 2011; Goldenberg and Levy 2009; Schaller and Latank 1995] also reported connection preference $r$ to be close to 1 in other online social networks (including Gowalla, Brightkite and Facebook). Even though they did not report the fractional dimension, from both the LiveJournal data in [Liben-Nowell et al. 2005] and our Renren data, it is reasonable to believe that the fractional dimension is also close to 1 . Therefore, empirical evidences all suggest that the real-world social networks indeed have connection preference close to the critical value and the network is navigable.

A natural question to ask next is how navigable networks naturally emerge? What are the forces that make the connection preference become close to the critical value? As Kleinberg pointed out in his survey paper [Kleinberg 2006] when talking about the above striking coincidence between theoretical prediction and empirical observation, "it suggests that there may be deeper phenomena yet to be discovered here". There are several studies trying to explain the emergence of navigable small-world networks [Mathias and Gopal 2001; Hu et al. 2011; Clauset and Moore 2003; Sandberg and Clarke 2006; Chaintreau et al. 2008], mostly by modeling certain underlying node or link dynamics (see additional related work below for more details).

In this paper, we tackle the problem in a novel way using a game-theoretic approach, which is reasonable in modeling individual behaviors in social networks without central coordination. One key insight we have is that connection preference $r$ is not a global preference but individual's own preference - some prefer to connect to more faraway nodes while others prefer to connect to nearby nodes. Therefore, we establish small-world formation games where individual node $u$ 's strategy is its own connection preference $r_{u}$ (Section 2). This game formulation is different from most existing network formation games where individuals' strategies are creating actual links in the network (c.f. [Tardos and Wexler 2007]). It allows us to directly explore the entire parameter space of connection preferences and answer the question on why nodes end up choosing a particular parameter setting leading to the navigable small world.

In terms of payoff functions, we first consider minimizing greedy routing distance to other nodes as the payoff, since it directly corresponds to the goal of navigable networks. However, Gulyás et al. [Gulyás et al. 2012] prove that with this payoff the navigable networks cannot emerge as a equilibrium for the one-dimensional case. Our empirical analysis also indicates that nodes will converge to random networks $\left(r_{u}=0, \forall u\right)$ rather than navigable networks for higher dimensions. Our empirical analysis further shows that if we adjust the payoff with a cost proportional to the grid distance of remote connections, the equilibria are sensitive to the cost factor.

The above unsuccessful attempt suggests that besides the goal of shortening distance to remote nodes, some other natural objective may be in play. Reciprocity is regarded as a basic mechanism that creates stable social relationships in a person's life [Gouldner 1960]. A number of prior works [Java et al. 2007; Liben-Nowell et al. 2005; Mislove et al. 2007] also suggest that people seek reciprocal relationships in online social networks. Therefore, we propose a payoff function that is the product of average distance of nodes to their long-range 
contacts and the probability of forming reciprocal relationship with long-range contacts. We call this game the distance-reciprocity balanced $(D R B)$ game. In practice, increasing relationship distance captures that individuals attempt to create social bridges by linking to "distant people", which can help them search for and obtain new resources. Meanwhile, increasing reciprocity captures that individuals look at social bonds by linking to "people like them", which could help them preserve or maintain resources. Therefore, the DRB game is natural since it captures sources of bridging and bonding social capital in building social integration and solidarity [Gittell and Vidal 1998]. We further allow heterogeneous utility functions in that different users may weigh the tradeoff between distance and reciprocity in different ways.

Even though the payoff function for the DRB game is very simple, our analysis demonstrates that it is extremely effective in producing navigable small-world networks as the equilibrium structure. In theoretical analysis (Section 3), we first show that navigable small world $\left(r_{u}=k, \forall u\right)$ and random small world $\left(r_{u}=0, \forall u\right)$ are the only two Nash equilibria of the DRB game, despite the flexible and heterogeneous utility functions. Moreover, for any strategy profile that is not the random network, it can always reach the navigable small world through a cascade of nearby nodes adopting strategy $k$ in a best-response dynamic.

In terms of the stability of NE, we prove that the navigable small world is a strong Nash equilibrium, which means that it tolerates collusion of any size trying to gain better payoff. Moreover, it also tolerates arbitrary deviations (without the objective of increasing anyone's payoff) of large groups of random deviators, since the system is guaranteed to return back to the navigable NE as soon as every node takes one best-response step. In contrast, random small world can be moved away from its equilibrium state by either a random perturbation of one node or a collusion of two nearby nodes, and when a small random set of nodes perturb to different strategies, we prove that the system is guaranteed to converge to the navigable small world as soon as every node takes one best-response step. Our theoretical analysis provides strong support that the navigable small-world NE is the unique and stable equilibrium that would naturally emerge in the DRB game.

We further examine the global function of social welfare (i.e., the total payoff of all nodes) and how selfish behavior of users affect the social welfare. Interestingly, we find that the global optimum can be reached by a fraction of nodes sacrificing their distance payoff to focus on reciprocity (by selecting a strategy greater than $k$ ) so that their neighbors could select strategy $k$ to reach a high balanced payoff of both distance and reciprocity. This situation reminds us social relationships generated by different social status (e.g. employeeemployer relationship) or by tight bonds with mutual understanding and support (such as marriages). Next we compare the social welfare of navigable and random small-world networks with the global optimum through the standard price of anarchy (PoA) and price of stability (PoS) metrics, which is the ratio of social welfare between the global optimum and the worst (or the best) Nash equilibrium, respectively. We show that navigable network has the better social welfare, and being only logarithmically worse than the global optimum.

To complement our theoretical analysis, we conduct empirical evaluations to cover more realistic game scenarios not covered by our theoretical analysis (Section 5). We first test random perturbation cases and show that arbitrary initial profiles always converge to the navigable equilibrium in a few steps, while a very small random perturbation (less than theoretical prediction) of the random small world causes it to quickly converge back to the navigable equilibrium. Next, we simulate more realistic scenarios where nodes have limited or no information about other nodes' strategies. We show that if they only learn their friends' strategies (with some noise), the system still converges close to the navigable equilibrium in a small number of steps. Further, even when the node has no information about other players' strategies and can only use its obtained payoff as feedback to search for the best strategy, the system still moves close to the navigable equilibrium within a few hundred steps (in the $100 \times 100$ grid). Finally we simulate the DRB game on Renren and LiveJournal 
networks, which have non-uniform population distributions different from Kleinberg's gridbased small-world model. Our simulation results show that in both networks, the game quickly converges to an equilibrium where connection preferences of users are close to the empirical ones.

In summary, our contributions are the following: (a) we propose the small-world formation game and design a balanced distance-reciprocity payoff function to explain the navigability of real social networks; (b) we conduct comprehensive theoretical and empirical analysis to demonstrate that navigable small world is the unique robust equilibrium that would naturally emerge from the game under both random perturbation and strategic collusions; and (c) our game reveals a new insight between distance, reciprocity and navigability in social networks, which may help future research in uncovering deeper phenomena in navigable social networks. To our best knowledge, this is the first game theoretic study on the emergence of navigable small-world networks, and the first study that linking relationship reciprocity with network navigability.

Additional related work. We provide additional details of prior works on explaining the emergence of navigable small-world networks, and other related studies not covered in the introduction.

Some studies try to explain navigability by assuming that nodes form links to optimize for a particular property. Mathias et al. [Mathias and Gopal 2001] assume that users try to make trade-off between wiring and connectivity. Hu et al. [Hu et al. 2011] assume that people try to maximize the entropy under a constraint on the total distances of their long-range contacts. These works rely on simulations to study the network dynamics. Moreover, the navigability of a network is sensitive to the weight of wiring cost or the distance constraint, and it is unlikely that navigable networks as defined by Kleinberg [Kleinberg 2002] would naturally emerge.

Another type of works propose node/link dynamics that converge to navigable smallworld networks. Clauset and Moore [Clauset and Moore 2003] propose a rewiring dynamic modeling a Web surfer such that if the surfer does not find what she wants in a few steps of greedy search, she would rewire her long-range contact to the current end node of the greedy search. They use simulations to demonstrate that a network close to Kleinberg's navigable small world will emerge after long enough rewiring rounds. Sandberg and Clarke [Sandberg and Clarke 2006] propose another rewiring dynamic where with an independent probability of $p$ each node on a greedy search path would rewire their long-range contacts to the search target, and provide a partial analysis and simulations showing that the dynamic converges to a network close to the navigable small world. Chaintreau et al. [Chaintreau et al. 2008] use a move-and-forget mobility model, in which a token starting from each node conducts a random walk (move) and may also go back to the starting point (forget), and use the distribution of the token on the grid as the distribution of the longrange contacts of the starting node. They provide theoretical analysis showing that there exists a critical forgetting value for which the move-and-forget model provides navigability. However, the underlying mechanism driving the critical value to be chosen in practice remains unclear.

The approach taken by these studies can be viewed as orthogonal and complementary to our approach: they aim at using natural dynamics (rewiring or mobility dynamics) to explain navigable small world, while we focus on directly exploring the entire parameter space of connection preferences of nodes and use game theoretic approach to show, both theoretically and empirically, that the nodes would naturally choose their connection preferences to form the navigable small world. The connection preference can be considered a higher level decision-making variable for individuals that pushes them to make long-range connections over time. In particular, selecting connection preference captures the people's process of cognitively creating behavioral plans (i.e., intensions) on how to distribute the 
finite time and effort among nodes of different distance. Once the preference is selected, the players would engage in activities such as rewiring or mobility dynamics to create long-range contacts with the corresponding connection intension. Moreover, all the prior studies only show that they converge approximately to the navigable small world, while in our game the navigable small world is precisely the only robust equilibrium. Finally, none of these works introduce reciprocity in their model and we are the first to link reciprocity with navigability of the small world.

Some studies use hyperbolic metric spaces or graphs to try to explain navigability in smallworld networks (e.g. [Boguñá et al. 2009; Papadopoulos et al. 2010; Krioukov et al. 2010; Krioukov et al. 2009; Chen et al. 2013; Gulyás et al. 2015]). However, they do not explain why connection preferences in real networks are around the critical value and how navigable networks naturally emerge. In particular, Chen et al. [Chen et al. 2013] show that the navigable small world in Kleinberg's model does not have good hyperbolicity. Most recently, Gulyás et al. [Gulyás et al. 2015] propose a game where each player tries to minimize the number of links in order to be able to greedily route to all other nodes. The equilibrium of the game is a scale-free network whose degree distribution follows a power law. However, this game is not intended and does not explain the emergence of navigable small-world network validated by Milgram and others' experiments, where greedy routing can be done efficiently in $O\left(\log ^{2} n\right)$ time in expectation, and relationship reciprocity is not included in any aspect of the game.

\section{SMALL-WORLD FORMATION GAMES}

In this section, we first present the game formulation based on Kleinberg's small-world model, and we then study the payoff function which is key to understanding the underlying mechanisms that give rise to navigable small world networks.

\subsection{Game Formulation based on Kleinberg's Small-World Model}

Small-world model. Let $V=\{(i, j): i, j \in[n]=\{1,2, \ldots, n\}\}$ be the set of $n^{2}$ nodes forming an $n \times n$ grid. For convenience, we consider the grid with wrap-around edges connecting the nodes on the two opposite sides, making it a torus. For any two nodes $u=\left(i_{u}, j_{u}\right)$ and $v=\left(i_{v}, j_{v}\right)$ on this wrap-around grid, the grid distance or Manhattan distance between $u$ and $v$ is defined as $d_{M}(u, v)=\min \left\{\left|i_{v}-i_{u}\right|, n-\left|i_{v}-i_{u}\right|\right\}+\min \left\{\mid j_{v}-\right.$ $\left.j_{u}|, n-| j_{v}-j_{u} \mid\right\}$.

The Kleinberg's small-world model has two universal constants $p, q \geq 1$, such that (a) each node has undirected edges connecting to all other nodes within lattice distance $p$, called its local contacts, and (b) each node has $q$ random directed edges connecting to possibly faraway nodes in the grid called its long-range contacts, drawn from the following distribution. Each node $u$ has a connection preference parameter $r_{u} \geq 0$, such that the $i$-th long-range edge from $u$ has endpoint $v$ with probability proportional to $1 / d_{M}(u, v)^{r_{u}}$, that is, with probability $p_{u}\left(v, r_{u}\right)=d_{M}(u, v)^{-r_{u}} / c\left(r_{u}\right)$, where $c\left(r_{u}\right)=\sum_{\forall v \neq u} d_{M}(u, v)^{-r_{u}}$ is the normalization constant. Let $\mathbf{r}$ be the vector of $r_{u}$ values on all nodes. We use $\mathbf{r} \equiv s$ to denote $r_{u}=s, \forall u \in V$.

The above model can be easily extended to $k$ dimensional grid (with wraparound) for any $k=1,2,3, \ldots$, where each long range contact is still established with probability proportional to $1 / d_{M}(u, v)^{r_{u}}$. We use $K(n, k, p, q, \mathbf{r})$ to refer to the class of Kleinberg random graphs with parameters $n, k, p, q$, and $\mathbf{r}$.

Small-world formation game. A game is described by a system of players, strategies and payoffs. Connection preference $r_{u}$ in Kleinberg's model reflects $u$ 's intention in establishing long-range contacts: When $r_{u}=0, u$ chooses its long-range contacts uniformly among all nodes in the grid; as $r_{u}$ increases, the long-range contacts of $u$ become increasingly clustered 
in its vicinity on the grid. Our insight is to treat connection preference as node's strategy in a game setting and study the game behavior.

More specifically, we model this via a non-cooperative game among nodes in the network. First, we assume that each $r_{u}$ is taken from a discrete set $\Sigma=\{0, \gamma, 2 \gamma, 3 \gamma, \ldots$,$\} , where \gamma$ represents the granularity of connection preference and is in the form of $1 / \mathrm{g}$ for some positive integer $g \geq 2$. Using discrete strategy set avoids nuances in continuous strategy space and is also reasonable in practice since people are unlikely to make infinitesimal changes.

Next, we model the small-world network formation as a game $\Gamma=\left(\Sigma, \pi_{u}\right)_{u \in V}$, where $V$ is the set of nodes (players) in the grid, connection preference $r_{u} \in \Sigma$ is the strategy of a player $u$, and $\pi_{u}: \mathcal{S} \rightarrow \mathbb{R}$ is the payoff function of $u$, with $\mathcal{S}=\Sigma \times \Sigma \times \ldots \times \Sigma$. An element $\mathbf{r}=\left(r_{1}, r_{2}, \ldots, r_{n}\right) \in \mathcal{S}$ is called a strategy profile.

Let $\mathcal{C}=2^{V} \backslash \emptyset$ denote the set of all coalitions. For each coalition $C \in \mathcal{C}$, let $-C=V \backslash C$, and if $C=\{u\}$, we denote $-C$ by $-u$. We also denote by $\mathcal{S}_{C}$ the set of strategies of players in coalition $C$, and $\mathbf{r}_{C}$ the partial strategy profile of $\mathbf{r}$ for nodes in $C$.

Objective. Greedy routing on the small-world network from a source node $u$ to a target node $v$ is a decentralized algorithm starting at node $u$, and at each step if routing reaches a node $w$, then $w$ selects one node from its local and long-range contacts that is closest to $v$ in grid distance as the next step in the routing path, until it reaches $v$. In [Kleinberg 2002], Kleinberg shows that given a two-dimensional grid, when $\mathbf{r} \equiv 2$, the expected number of greedy routing steps (called delivery time) is $O\left(\log ^{2} n\right.$ ), but when $\mathbf{r} \equiv s \neq 2$, it is $\Omega\left(n^{c}\right)$ for some constant $c$ related to $s$. More generally, for any $k$ dimensional grid, it is shown that $\mathbf{r} \equiv k$ is the critical value allowing efficient greedy routing. Hence, we call Kleinberg's small world with $\mathbf{r} \equiv k$ the navigable small world.

Interestingly, empirical evidences have demonstrated that the real-world network is navigable with the connection preference close to the critical value [Liben-Nowell et al. 2005; Cho et al. 2011; Adamic and Adar 2005; Goldenberg and Levy 2009; Lambiotte et al. 2008; Illenberger et al. 2013]. We aim to explain this striking coincidence from the perspective of individual incentives. In particular, our objective is to study intuitively appealing payoff functions $\pi_{u}$ and find one that individual efforts to get this payoff lead fairly quickly to the emergence of navigable small-world network.

\subsection{Routing-based Payoff}

As navigable small world achieves best greedy routing efficiency, it is natural to consider the payoff function as the expected delivery time to the target in greedy routing. Given the strategy profile $\mathbf{r} \in \mathcal{S}$, let $t_{u v}\left(r_{u}, \mathbf{r}_{-u}\right)$ be the expected delivery time from source $u$ to target $v$ via greedy routing. The payoff function is given by:

$$
\pi_{u}\left(r_{u}, \mathbf{r}_{-\mathbf{u}}\right)=-\sum_{\forall v \neq u} t_{u v}\left(r_{u}, \mathbf{r}_{-u}\right) .
$$

We take a negation on the sum of expected delivery time because nodes prefer shorter delivery time.

Although the above payoff function is intuitive and simple, it has some serious issues. Prior work [Gulyás et al. 2012] has already proved that, with the length of greedy paths as the payoff, player u's best response is to link uniformly (i.e., $r_{u}=0$ ) for the one-dimensional case. For higher dimensions, Figure 3 shows the expected delivery time for a single node $u$ at a $100 \times 100$ grids, where each node generates $q=10$ links. We see that when other nodes fixed their strategy (e.g., $\mathbf{r}_{-u} \equiv 2$ ), the best strategy of a single node $u$ is 0 . More tests on different initial conditions reach the same result that the system will converge to the random small-world networks. The intuitive reason is that to reach other nodes quickly, it is better for a node to evenly spread its long-range contacts from the individual prospective 


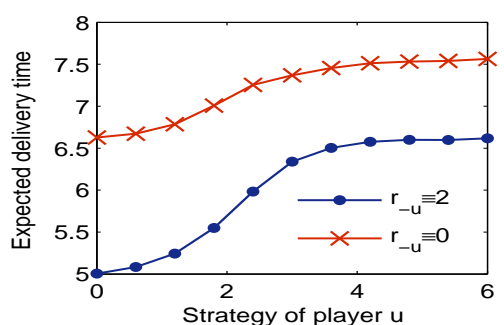

Fig. 3. The expected delivery time for a player $u$ with different strategies.

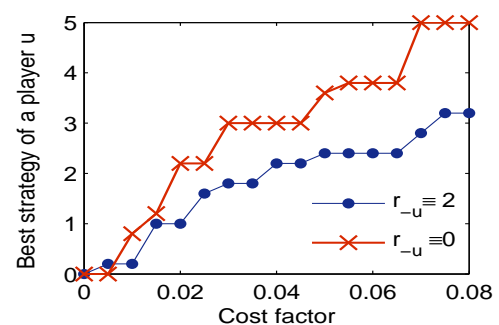

Fig. 4. The best response of a player $u$ given different cost factors.

(or equivalently, seeking the long-range contacts of the largest distance on average given $\left.r_{u} \geq 0\right)$. This is inconsistent with empirical evidence that real-world networks are navigable ones, where links are much more likely to connect neighbor nodes than distant nodes.

In practice, creating and maintaining long-range links have higher costs, so one may adapt the above payoff function by adding the grid distances of long-range contacts as a cost term in the payoff function:

$$
\pi_{u}\left(r_{u}, \mathbf{r}_{-\mathbf{u}}\right)=-\sum_{\forall v \neq u} t_{u v}\left(r_{u}, \mathbf{r}_{-u}\right)-\lambda \sum_{\forall v \neq u} p_{u}\left(v, r_{u}\right) d_{M}(u, v),
$$

where $\lambda$ is a factor controlling the long range cost and $p_{u}\left(v, r_{u}\right)=d_{M}(u, v)^{-r_{u}} / c\left(r_{u}\right)$ is the probability that $u$ takes $v$ as a long-range contact under the strategy of $r_{u}$. A larger $\lambda$ means users are more concerned with distance costs. Figure 4 shows that the best strategy of a user $u$ is significantly influenced by the cost factor. Similar result is also shown in [Gulyás et al. 2012]. Thus, it is unclear if the navigable small-world network can naturally emerge from this type of game.

In the above payoff functions, we use the expected delivery time to measure the routing efficiency to an arbitrary node. It is also possible to give more complex payoff functions by considering the distribution functions of delivery time, such as the percentage of nodes that can be delivered within a given number of steps. However, given that individuals can have different strategies, it is very difficult to obtain the explicit form of delivery time $t_{u v}\left(r_{u}, \mathbf{r}_{-u}\right)$ in terms of users strategies $\mathbf{r}$. Due to this disadvantage of the delivery time-based games, there is no theoretical guarantee that the network formation would converge to the desired navigable small world.

\subsection{Distance-Reciprocity Balanced Payoff}

The previous section demonstrates that seeking short routing distance alone cannot explain the emergence of navigable small world, and thus people in the social network must have some other objective to achieve. Reciprocity is regarded as a basic mechanism that creates stable social relationships in the real world [Gouldner 1960]. Several empirical studies [Java et al. 2007; Liben-Nowell et al. 2005; Mislove et al. 2007] also show that high reciprocity is also a typical feature present in real small-world networks (such as Flickr, YouTube, LiveJournal, Orkut and Twitter).

Therefore, we consider the payoff of a user $u$ as the following balanced objective between distance and reciprocity:

$$
\pi_{u}\left(r_{u}, \mathbf{r}_{-\mathbf{u}}\right)=\left(\sum_{\forall v \neq u} p_{u}\left(v, r_{u}\right) d_{M}(u, v)\right)^{\alpha_{u}} \times\left(\sum_{\forall v \neq u} p_{u}\left(v, r_{u}\right) p_{v}\left(u, r_{v}\right)\right)
$$


where $\sum_{\forall v \neq u} p_{u}\left(v, r_{u}\right) d_{M}(u, v)$ is the mean grid distance of $u$ 's long-range contacts, $\sum_{\forall v \neq u} p_{u}\left(v, r_{u}\right) p_{v}\left(u, r_{v}\right)$ is the mean probability for $u$ to form bi-directional links with its long-range contacts, i.e., reciprocity, and $\alpha_{u}\left(\alpha_{u}>0\right)$ is a constant exponent with respect to node $u$, capturing how that user weighs the relative importance of distance and reciprocity. Note here the tradeoff exponent $\alpha_{u}$ could be heterogeneous among players, modeling users having different weights on the balance between the distance and reciprocity tradeoff. So our utility function is very flexible and actually represents a large class of tradeoff functions. We refer the small-world formation game with payoff function in Eq.(3) the Distance-Reciprocity Balanced (DRB) game.

The payoff function in Eq.(3) reflects two natural objectives users in a social network want to achieve: first, they want to connect to remote nodes, which may give them diverse information as in the famous "the strength of weak ties argument" by Granovetter [Granovetter 1973]; second, they want to establish reciprocal relationship which are more stable in the long term. However, these two objectives can be in conflict for a node $u$ when others prefer linking in their vicinity (i.e., other nodes $v$ choosing positive exponent $r_{v}$ ). In this case, faraway long-range contacts are less likely to create reciprocal links. Therefore, node $u$ should obtain the maximum payoff when it achieves a balance between the two objectives. We use the simple product of distance and reciprocity objectives to model this tradeoff, and allow different nodes to have different emphasis on distance-reciprocity tradeoffs with their own exponents. One may also consider the addition of the distance term and the reciprocity term to model the tradeoff, but since the two quantities have different unit of scale - distance scales from 1 to $O(k n)$ while reciprocity is a probability between 0 and 1 , we believe the multiplicative formulation makes more sense.

We remark that the reciprocity term $\sum_{\forall v \neq u} p_{u}\left(v, r_{u}\right) p_{v}\left(u, r_{v}\right)$ does not consider reciprocity formed by fixed local contacts. Effectively, we disregard local contacts and treat $p=0$ in the small world setting $K(n, k, p, q, \mathbf{r})$. This treatment makes our analysis more streamlined and only focused on long-range contacts, and it also makes intuitive sense: the local contacts are passively given based on geographic location, while long-range contacts are actively established by nodes based on their connection preference, and thus reciprocity based on long-range contacts could make more sense. For example, your neighbors in the same apartment building are your local contacts by physical location, but it does not mean that they are your friends, and you still need to intentionally establish friendship (based on your preference) among your neighbors, and thus reciprocity only by physical location does not mean much but reciprocity based on actively established relationship does mean a lot for an individual.

Existing network formation games typically use pure-link-based strategy and lead to mostly trivial equilibria such as cliques or stars. Different from prior games, we use the link probability functions as the strategies, which can be viewed as a mix strategy on pure links, but with restricted distributions. Here, we focus on the power-law distributions assumed in Kleinberg's small-world models, which is also supported from findings in several real complex networks, such as human travel network [Gonzlez et al. 2008; Zhao et al. 2015], communication network [Krings et al. 2009], trade network [Bhattacharya et al. 2008] and other social networks [Liben-Nowell et al. 2005; Cho et al. 2011].

In practice, the strategy captures certain behavioral preference of players related to connection. One concrete example is mobility preference in human travel network, where the link distribution can be understood as the trip distance distribution by taking the grid location of a node as its home. In this network, the strategy $r_{u}$ captures the mobility preference of individual $u$, large $r_{u}$ results in large possibility of short distance travel. Our game means that each user adjusts its mobility preference to the heterogeneous preferences of others for a better payoff, such as obtaining non-redundant information via long-distance visits and social support enforced by mutual visits. 


\section{PROPERTIES OF THE DRB GAME}

In this section, we conduct theoretical analysis to discover the properties of the DRB game. We begin by considering the problem of the existence of equilibria in the game, and if the answer is yes, whether there exist multiple equilibria. In Section 3.1, we prove that DRB game has only two Nash equilibria $\mathbf{r} \equiv k$ and $\mathbf{r} \equiv 0$, corresponding to the navigable and random small-world networks, respectively. Given multiple Nash equilibria, we further investigate if the navigable small world possesses further properties making it the likely choice in practice. This is the task of the next two sections.

One way to solve the problem of multiple equilibria is to consider a more appealing equilibrium concept-strong Nash equilibrium (SNE). While in a NE no player can improve its payoff by unilateral deviation, in a SNE there is no coalition of players that can improve their payoffs by collective deviation. In Section 3.2, we show that the navigable small-world equilibrium is a SNE in the game, which is much more stable than the random small-world equilibrium.

Another way to approach the problem is to study the convergence to equilibrium under the best response dynamics. This dynamics could help to select among multiple equilibria of the game. In Section 3.3, we show that the navigable small-world equilibrium is reachable via best response dynamics from any state not in the other equilibrium. We also prove that the navigable small-world NE can also tolerate large perturbations of players under best response dynamics, whereas the random small-world NE is extremely unstable under perturbation.

We finally give a description how the navigable small-world network is formed by summarizing our results in Section 3.4.

\subsection{Equilibrium Existence}

Nash equilibrium (NE) for the strategic game $\Gamma=\left(\Sigma, \pi_{u}\right)_{u \in V}$ is a strategy profile $\mathbf{r}^{*} \in \mathcal{S}$ such that each player's strategy $r_{u}^{*}(\forall u \in V)$ is a best response to the other players' strategies $\mathbf{s}_{-u}^{*}$, where the best response is defined as follow:

Definition 3.1 (Best response). Player $u$ 's strategy $r_{u}^{*} \in \Sigma$ is a best response to the strategy profile $\mathbf{r}_{-\mathbf{u}} \in \mathcal{S}_{-u}$ if

$$
\pi_{u}\left(r_{u}^{*}, \mathbf{r}_{-\mathbf{u}}\right) \geq \pi_{u}\left(r_{u}, \mathbf{r}_{-\mathbf{u}}\right), \forall r_{u} \in \Sigma \backslash\left\{r_{u}^{*}\right\}
$$

Moreover, if " $\geq$ " above is actually " $>$ " for all $r_{u} \neq r_{u}^{*}$, then $s_{u}^{*}$ is the unique best response to $\mathbf{s}_{-\mathbf{u}}$. We denote this unique best response as $B_{u}\left(\mathbf{s}_{-u}\right)$. Strategy profile $\mathbf{r}^{*}$ is a strict Nash equilibrium if for every player $u \in V, r_{u}^{*}$ is the unique best response to $\mathbf{r}_{-u}^{*}$.

We first show that the navigable small-world network is a Nash Equlibrium of the DRB game. To do so, we focus on a local region centering around a node $w$ preferring local connection, and we have the following important lemma.

LEMMA 3.1. In the $k$-dimensional DRB game, for any constant $\delta$, there exists $n_{0} \in \mathbb{N}$ (may depend on $\delta$ ), for any $n \geq n_{0}$, for any non-zero strategy profile $\mathbf{r} \not \equiv 0$, if a node $w$ satisfies $r_{w} \geq k$ or $r_{w}=\max _{v \in V} r_{v}$, then for any node $u$ within $\delta$ grid distance of $w$ (i.e. $\left.d_{M}(u, w) \leq \bar{\delta}\right)$, u has the unique best response of $r_{u}=k$.

Proof (Sketch). The intuition is as follows. When a node $w$ satisfying $r_{w} \geq k$ or $r_{w}=\max _{v \in V} r_{v}$, it prefers its long-range contacts to be in its vicinity. For a nearby node $u$ with $d_{M}(u, v) \leq \delta$, the case of $r_{u}=k$ provides the best balance between good grid distance to long-range contacts and high reciprocity (even just counting the reciprocity received from $w)$. In other cases, the node $u$ obtains either too low reciprocity or too short average grid distance to long-range contacts. In the case of $r_{u}<k$, the node $u$ could increase the average grid distance to long-range contacts by an factor of $O(\ln n)$, but the reciprocity can be reduced by a factor of $\Omega\left(n^{\gamma}\right)$, as compared with those provided by $r_{u}=k$. Thus, the ratio 
of payoff for $r_{u}<k$ to payoff for $r_{u}=k$ is at most $O\left(\ln ^{\alpha_{u}} n / n^{\gamma}\right)$, which is smaller than one given sufficient large $n$. Similarly, in the case of $r_{u}>k$, the node $u$ could increase the reciprocity by an factor of $O(\ln n)$, but the average grid distance to long-range contacts can be reduced by a factor of $\Omega\left(n^{\gamma}\right)$, as compared with those provided by $r_{u}=k$. Thus, the ratio of payoff for $r_{u}>k$ to payoff for $r_{u}=k$ is also at most $O\left(\ln n / n^{\alpha_{u} \gamma}\right)$, which is also smaller than one given sufficient large $n$. The detailed proof is included in Appendix B.

The above lemma shows that given a non-zero profile, we can find a local region where the best response of every node is $k$. This lemma is instrumental to several analytical results, including the following theorem.

THEOREM 3.1. For the DRB game in a $k$-dimensional grid, the following is true for sufficiently large $n:{ }^{1}$ For every node $u \in V$, every strategy profile $\mathbf{r}$, and every $s \in \Sigma$, if $\mathbf{r}_{-u} \equiv s$, then $u$ has a unique best response to $\mathbf{r}_{-u} \equiv s$ :

$$
B_{u}\left(\mathbf{r}_{-u} \equiv s\right)= \begin{cases}k & \text { if } s>0, \\ 0 & \text { if } s=0 .\end{cases}
$$

Proof (Sketch). For the case of $s>0$, given the strategy profile of $\mathbf{r}_{-u} \equiv s$, for every node $u$, each of its nearest neighbor $w$ (i.e., $d_{M}(u, w)=1$ ) satisfies $r_{w}=\max _{v \in V} r_{v}$. Thus by Lemma 3.1, node $u$ 's unique best response to $\mathbf{r}_{-u} \equiv s$ is $r_{u}=k$.

When $s=0$, all others nodes link uniformly. In this case, the reciprocity for node $u$ becomes a constant independent of its strategy $r_{u}$. Thus, $r_{u}$ should be selected to maximize average distance of $u$ 's long-range contacts, which leads to $r_{u}=0$. The detailed proof of this case is included in Appendix C.

Theorem 3.1 shows that when all other nodes use the same nonzero strategy $s$, it is strictly better for $u$ to use strategy $k$; when all other nodes uniformly use the 0 strategy, it is strictly better for $u$ to also use 0 strategy. When setting $s=k$ and $s=0$, we have:

Corollary 3.1. For the DRB game in the $k$-dimensional grid, the navigable smallworld network $(\mathbf{r} \equiv k)$ and the random small-world network $(\mathbf{r} \equiv 0)$ are the two strict Nash equilibria for sufficiently large $n$, and there are no other uniform Nash equilibria.

We next examine if there exists any non-uniform equilibrium.

THEOREM 3.2. In the $k$-dimensional DRB game, there is no non-uniform Nash equilibrium for sufficiently large $n$.

ProOF. Given any non-uniform strategy profile $\mathbf{r}$, let $V_{\geq k}=\left\{v \mid r_{v} \geq k\right\}$. If $V_{\geq k} \neq \emptyset$, we can find a pair of grid neighbors $(u, w)$ with $r_{u} \neq k$ and $r_{w} \geq k$. If $V_{\geq k}=\emptyset$, we can find a pair of grid neighbors $(u, w)$ with $r_{u} \neq k$ and $r_{w}=\max _{v \in V} r_{v}$. In either case, we know the node $u$ could obtain better payoff by unilaterally deviating to the strategy $r_{u}=k$ by Lemma 3.1. Therefore, non-uniform strategy profile $\mathbf{r}$ is not a Nash equilibrium.

Combining the above theorem with Corollary 3.1, we see that DRB game has only two Nash equilibria $\mathbf{r} \equiv k$ and $\mathbf{r} \equiv 0$, corresponding to the navigable and random small-world networks, respectively.

\subsection{Equilibrium Stability under Collusion}

While in an NE no player can improve its payoff by unilateral deviation, some of the players may benefit (sometimes substantially) from forming alliances/coalitions with other players.

\footnotetext{
${ }^{1}$ Technically, a statement being true for sufficiently large $n$ means that there exists a constant $n_{0} \in \mathbb{N}$ that may only depend on model constants such as $k, \gamma$ and $\alpha_{u}$, such that for all $n \geq n_{0}$ the statement is true in the grid with parameter $n$.
} 
So we study a more general $t$-Strong Nash equilibrium ( $t$-SNE) to study the resilience to coalitions.

Definition 3.2 ( $t$-Strong Nash equilibrium). For a number $t \in\{1,2, \ldots,|V|\}$, a strategy profile $\mathbf{r}^{*} \in \mathcal{S}$ is a $t$-strong Nash equilibrium if for all $C \in \mathcal{C}$ with $|C| \leq t$, there does not exist any $r_{C} \in \mathcal{S}_{C}$ such that

$$
\forall u \in C, \pi_{u}\left(\mathbf{r}_{C}, \mathbf{r}_{-C}^{*}\right) \geq \pi_{u}\left(\mathbf{r}^{*}\right), \exists u \in C, \pi_{u}\left(\mathbf{r}_{C}, \mathbf{r}_{-C}^{*}\right)>\pi_{u}\left(\mathbf{r}^{*}\right) .
$$

When $t=|V|$, we simply call $\mathbf{r}^{*}$ the strong Nash equilibrium (SNE). Note that 1-SNE falls back to NE.

We first show the important result that the navigable small-world network is able to tolerate collusion of any group of players, i.e., $\mathbf{r} \equiv k$ is a $|V|$-SNE or simply SNE.

THEOREM 3.3. For the DRB game in the $k$-dimensional grid, the navigable small-world network $(\mathbf{r} \equiv k)$ is a strong Nash equilibrium for sufficiently large $n$.

Proof (SKeTCh). We prove a slightly stronger result - any node $u$ in any strategy profile $\mathbf{r}$ with $r_{u} \neq k$ has strictly worse payoff than its payoff in the navigable small world. Intuitively, when $u$ deviates to $0 \leq r_{u}<k$, its loss on reciprocity would outweigh its gain on link distance; when $u$ deviates to $r_{u}>k$, its loss on link distance is too much to compensate any possible gain on reciprocity. The detailed proof is in Appendix D.

The above theorem shows that the navigable small-world equilibrium is not only immune to unilateral deviations, but also to deviations by coalitions of any size, and in particular it is Pareto-optimal, such that no player can improve her payoff without decreasing the payoff of someone else.

After showing that the navigable small-world is robust to collusions of any size, we now show that random small world equilibrium is not stable even under the collusion of a pair of nodes.

TheOREM 3.4. For the DRB game in a k-dimensional grid, the random small-world $N E \mathbf{r} \equiv 0$ is not a 2-strong Nash equilibrium for sufficiently large $n$.

Proof (SKeTCH). If a pair of grid neighbors collude to deviate their strategies to $k$, they could gain much benefit in terms of reciprocity, as compared with the loss of relationship distance. As a result, they would both get better payoff than their payoff in $\mathbf{r} \equiv 0$. The detailed proof is in Appendix E.

\subsection{Convergence under Best Response Dynamics}

For our game, we finally study its best response dynamics to investigate its properties of convergence to Nash equilibria. Best response dynamics are typically specified in terms of asynchronous steps: in each asynchronous step, one player moves from its current strategy to its best response to the current strategy profile, and thus the entire strategy profile moves one step accordingly. To facilitate the study of convergence speed, we also look into synchronous steps for the best response dynamics: in each synchronous step, every player moves from its current strategy to its best response to the current strategy profile, and collectively we count this as one synchronous step.

With the concept of best-response dynamics, we first show that for any non-zero profile, we can find a node that triggers a cascade of adopting strategy $k$ from neighbors to neighbors of neighbors, and so on, ultimately leading to the navigable small world equilibrium.

THEOREM 3.5. In the $k$-dimensional DRB game, for sufficiently large $n$, the navigable small-world equilibrium $\mathbf{r} \equiv k$ is reachable via best response dynamics from any non-zero strategy profile $\mathbf{r} \not \equiv 0$. Moreover, if all nodes move synchronously in the best response dy- 
namics, then it takes at most $k\lfloor n / 2\rfloor$ synchronous steps for any non-zero strategy profile to converge to the navigable small-world equilibrium $\mathbf{r} \equiv k$.

Proof. Let $V_{w}(j)=\left\{v \mid d_{M}(v, w) \leq j\right\}$. Given a non-zero profile $\mathbf{r}$, we can find a node $w$ satisfying $r_{w} \geq k$ or $r_{w}=\max _{v \in V} r_{v}$. Given a constant $\delta(\delta \geq 2)$, Lemma 3.1 implies that for sufficiently large $n$, for every $u \in V_{w}(\delta)$, in one asynchronous step $u$ will set $r_{u}=k$. Then consider $u$ 's neighbors $V_{u}(\delta)$, in one asynchronous step each of them will also set their strategy to $k$. Following this cascade it is clear that there exists a step sequence such that the non-zero profile $\mathbf{r}$ will reach the navigable small world $\mathbf{r} \equiv k$.

We now consider that all nodes move synchronously. Again we first find a node $w$ satisfying $r_{w} \geq k$ or $r_{w}=\max _{v \in V} r_{v}$. By Lemma 3.1 all nodes in $V_{w}(\delta / 2)(\delta / 2 \geq 1)$ move to strategy $k$ in the first synchronous step. Consider the second synchronous step. Even though we are not sure if node $w$ adopts strategy $k$ in the first synchronous step, we know that $w$ adopts $k$ in the second synchronous step since $w$ has neighbors adopting $k$ after the first synchronous step. Moreover, for all nodes in $V_{w}(\delta / 2)$, their mutual grid distance is at most $\delta$, and thus Lemma 3.1 applies to these nodes in the second synchronous step and they all stay at strategy $k$. Finally for their grid neighbors within grid distance $\delta / 2$, essentially nodes in $V_{w}(\delta) \backslash V_{w}(\delta / 2)$, they will also adopt strategy $k$ in the second synchronous step. Repeating the above procedure, all nodes that have adopted $k$ will keep $k$ while their grid neighbors will also adopt $k$. Since the longest grid distance among nodes in the $k$-dimension grid is $k\lfloor n / 2\rfloor$, after at most $k\lfloor n / 2\rfloor$ synchronous steps, all nodes adopt $k$.

The proof of the above theorem provides valuable insights into the scalability of the game. Notice that a $k$-dimension grid contains a total of $|V|=n^{k}$ players, so the above theorem states that, for any non-zero strategy profile, the convergence time to navigable NE is at most $O\left(|V|^{\frac{1}{k}}\right)$ synchronous steps if players move synchronously in the best response dynamics. Also, any player $u$ involved in the cascade of adopting $r_{u}=k$ can make this best decision locally according to the strategies of the players in his neighborhood. Thus our game is scalable with the number of players.

Next, we would like to see if the navigable equilibrium can also tolerate perturbations of players under best response dynamics, where the perturbations could be arbitrary and there is no guarantee that perturbed players are better off. From Theorem 3.5, we know that as long as not all nodes deviate to zero, there exists a best response dynamic sequence for the system to go back to the navigable small world, and if all nodes move synchronously, the system reaches the navigable small world in at most $k\lfloor n / 2\rfloor$ synchronous steps. We now give a further result on the stability of navigable small-world in tolerating perturbations of random players: we show that even if each individual independently perturbs to an arbitrary strategy with a fairly large probability, the system moves back to the navigable small world in just one synchronous step, and even if players move asynchronously, it is guaranteed that the system moves back to the navigable small world after each node takes at least one asynchronous step.

THEOREM 3.6. Consider the navigable small-world equilibrium $\mathbf{r} \equiv k$ for the DRB game in a $k$-dimensional grid $(k>1)$. Suppose that with probability $p_{u}$ each node $u \in V$ independently perturbs $r_{u}$ to an arbitrary strategy $r_{u}^{\prime} \in \Sigma$, and with probability $1-p_{u} r_{u}^{\prime}=r_{u}$. Let $\alpha_{\text {min }}=\min _{u \in V} \alpha_{u}$, then for any constant $\varepsilon$ with $0<\varepsilon<\min \left\{1, \alpha_{\min }\right\} \gamma / 4$, there exists $n_{0} \in \mathbb{N}$ (depending only on $k, \gamma$, and $\varepsilon$ ), for all $n \geq n_{0}$, if $p_{u} \leq 1-n^{-\varepsilon}$, with probability at least $1-1 / n$, the perturbed strategy profile $\mathbf{r}^{\prime}$ moves back to the navigable small world $(\mathbf{r} \equiv k)$ in one synchronous step, or as soon as every node takes at least one asynchronous step in the best response dynamics.

Proof (Sketch). The independently selected deviation node set satisfies that with high probability, for any node $u$, at sufficiently many distance levels from $u$ there are enough 
fraction of non-deviating nodes. We then show that $u$ obtains higher order payoff just from these non-deviating nodes than any possible payoff she could get from any possible deviation. The detailed proof is in Appendix F.

Notice that the bound of $1-n^{-\varepsilon}$ is close to 1 when $n$ is sufficiently large, meaning that the navigable equilibrium tolerates arbitrary deviations from a large number of random nodes.

For the random small-world network, which is shown to be the other NE, Theorem 3.5 already implies that even one deviating player could possibly drive the system out of the random small-world equilibrium and lead it towards the navigable small-world equilibrium. However, converging to navigable small world is not guaranteed in this case. In the following, we show a stronger convergence result: if each individual $u$ deviates from $r_{u}=0$ independently with even a small probability, then the system could switch to the navigable small world in just one synchronous step, or after each node takes at least one asynchronous step, and the convergence to the navigable small world is guaranteed in this case.

TheOREM 3.7. For the DRB game in a $k$-dimensional grid $(k>1)$ with the initial strategy profile $\mathbf{r} \equiv 0$ and a finite perturbed strategy set $S \subset \Sigma$ with at least one non-zero entry $(0<\max S \leq \beta)$, for any constant $\varepsilon$ with $0<\varepsilon<\gamma / 2$, there exists $n_{0} \in \mathbb{N}$ (depending only on $k, \gamma$, and $\varepsilon$ ), for all $n \geq n_{0}$, if for any $u \in V$, with independent probability of $p \geq n^{-\frac{(k-1) \varepsilon}{k+\beta}}, r_{u} \in S \backslash\{0\}$ after the perturbation, then with probability at least $1-1 / n$, the network converges to the navigable small world in one synchronous step, or as soon as every node takes at least one asynchronous step in the best response dynamics.

Proof (SKeTCH). We consider the gain of a node $u$ when selecting $r_{u}=k$ separately from each group of nodes with the same strategy after the perturbation, and then apply the results in Theorem 3.1. The full proof is in Appendix G.

Note that $1 / n^{\frac{(k-1) \varepsilon}{k+\beta}}$ is very small for large $n$ and a finite perturbed strategy set $S$, which implies that the best response of any node $u$ in the perturbed profile becomes $r_{u}=k$ as long as a small number of random nodes are perturbed to a finite set of nonzero strategies.

\subsection{Implications from Theoretical Analysis}

Combining the above theorems together, we obtain a better understanding of how the navigable small-world network is formed. From any arbitrary initial state, best response dynamic drives the system toward some equilibrium, with the navigable small world as one of them (Corollary 3.1 and Theorem 3.5). Even if the systems temporarily converges to a non-navigable equilibrium, the state will not be stable - either a small-size collusion (Theorem 3.4) or a small-size random perturbation (Theorem 3.7) would make the system leave the current equilibrium and quickly enter the navigable equilibrium. Once entering the navigable equilibrium, it is very hard for the system to move away from it - no collusion of any size would drive the system away from this equilibrium (Theorem 3.3), and even if a large random portion of nodes deviate arbitrarily the system still converge back to the navigable equilibrium as long as each node takes one best-response step (Theorem 3.6). These theoretical results strongly support that the navigable small world is the unique stable system state, which suggests that the fundamental balance between reaching out to remote people and seeking reciprocal relationship is crucial to the emergence of navigable small-world networks.

\section{QUALITY OF EQUILIBRIA}

In a Nash equilibrium, each user is maximizing its individual payoff. However, there is also a global function of social welfare, which is the total payoff of all nodes. A natural question then is how the social welfare of a system is affected when its users are selfish. Thus, in this 
section, we would like to examine how good the solution represented by an equilibrium is relative to the global optimum.

To study the social welfare, we focus on the homogenous network in which all players use the same tradeoff exponent $\alpha$, since it is difficult to normalize and integrate individual utility measures if they have different emphasis on distance or reciprocity. We first examine the global optimum.

THEOREM 4.1. In the $k$-dimensional homogeneous DRB game, the optimal social welfare is $\Theta\left(\frac{n^{\alpha+k}}{\ln ^{\alpha+1} n}\right)$ for sufficiently large $n$.

Proof (Sketch). We prove that a node $u$ with $r_{u}=k$ could get high payoff if it has at least one neighbor $v$ with $r_{v}>k$. In this case, the node $u$ could get both large grid distance to long-range contacts and high reciprocity (at least from $v$ ). So if the system has a constant fraction of such nodes, the social welfare is optimized. The detailed proof is included in Appendix H.

The proof of the above theorem provides some interesting insights: First, the optimal strategy profile is not the navigable network where all players get the same payoff, instead, it exhibits inequality in the distribution of payoff. The rich (e.g., those with strategy of $k$ ) could get high payoff whereas the poor (e.g., those with strategy larger than $k$ ) only get very low payoff. Furthermore, the optimum social welfare is achieved when the poor sacrifice their distance payoff and focus on their reciprocity (by selecting a strategy greater than $k$ ), so that their rich neighbors could obtain a high balanced payoff of both distance and reciprocity. This situation reminds us social relationships generated by different social status (e.g. employee-employer relationship) or by tight bonds with mutual understanding and support (such as marriage relationship).

We next focus on the standard measures of the sub-optimality introduced by selfinterested behavior. In particular, price of stability (PoS) is the ratio of the solution quality at the best Nash equilibrium relative to the global optimum, whereas the price of anarchy (PoA) is the ratio of the worst Nash equilibrium to the optimum.

THEOREM 4.2. In the $k$-dimensional homogeneous DRB game, for sufficiently large $n$, the PoS is $\Theta(\ln n)$ and the PoA is $\Theta\left(\frac{n^{k}}{\ln ^{\alpha+1} n}\right)$.

Proof (Sketch). From the analysis in Section 3.1, we know that the system has only two Nash equilibria $\mathbf{r} \equiv k$ and $\mathbf{r} \equiv 0$, corresponding to navigable and random small-world networks, respectively. We show that the navigable small-world NE is a better equilibrium since the strategy of $k$ provides the best balance between grid distance to long-range contacts and reciprocity. Combined with Theorem 4.1, we get the PoS and PoA of the system. The detailed proof is included in Appendix I.

The above theorem indicates that, in the good case when the system is in the navigable network equilibrium, the social welfare is reasonably close to the social optimum (with ratio $\Theta(\ln n)$ among $n^{k}$ nodes), but in the bad case when the network is in the random network equilibrium, the social welfare is far from the social optimum.

\section{EMPIRICAL EVALUATION}

In this section, we empirically examine the stability of navigable small-world NE. We simulate the DRB game on two dimensional grids, and consider nodes having full information, limited information, or no information of other players' strategies.

In Section 5.1 and Section 5.2, we focus on the homogeneous game $\left(\alpha_{u}=1, \forall u \in V\right)$ as our equilibrium analysis is robust to $\alpha_{u}$ under the $k$-dimensional grid of people. In Section 5.3 , we further examine the heterogeneous game under non-uniform population density 


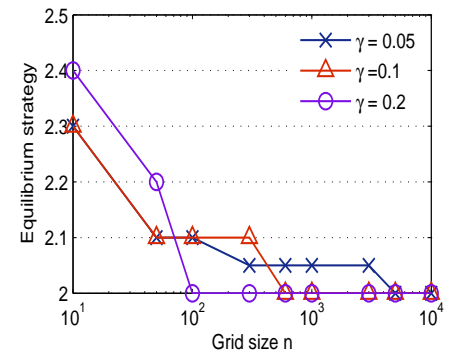

Fig. 5. Equilibrium strategy in the 2D grid of different size and granularity.

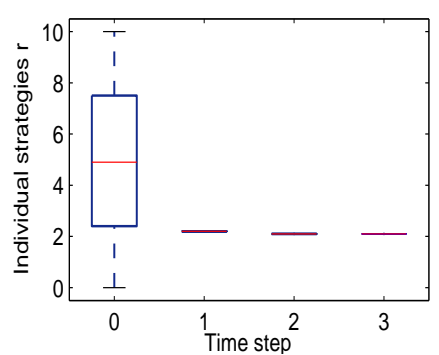

Fig. 6. The return to navigable small-world NE (perturbed probability $\mathrm{p}=1$ ).

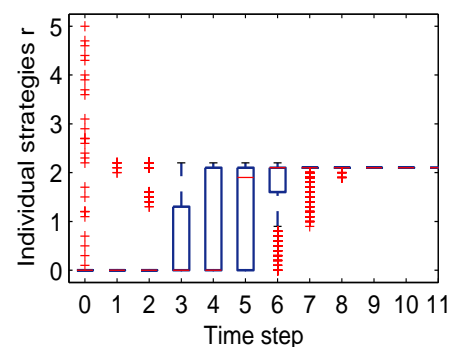

Fig. 7. From random NE to smallworld NE (perturbed probability $\mathrm{p}=0.01)$.

across real social networks. Before the main empirical evaluation, we first test the effect of the grid size $n$ on navigable equilibrium, since our theoretical results require a sufficiently large $n$.

Our theoretical analysis shows that one can find a large enough constant $n_{0}$, such that the navigable equilibrium is exactly $\mathbf{r} \equiv 2$ for all $n \geq n_{0}$. Thus, we first verify empirically the relationship between the size of the grid and the actual connection preference value for the equilibrium. Figure 5 shows how the equilibrium value changes over $n$ in a $2 \mathrm{D}$ grid, with various granularity. For example, with a granularity of $\gamma=0.1$, the equilibrium decreases from $\mathbf{r} \equiv 2.3$ for a very small $10 \times 10$ grid, to $\mathbf{r} \equiv 2$ for a $1000 \times 1000$ grid. This shows that we do not need a very large grid in order to obtain results close to our theoretical predictions. In our following experiments, we use a $100 \times 100$ grid with the granularity $\gamma=0.1$, which leads to an equilibrium $\mathbf{r} \equiv 2.1$ close to theoretical prediction while reducing the simulation cost.

\subsection{Stability of NE under Perturbation}

To demonstrate the stability of navigable NE, we simulate the DRB game with random perturbation. At time step 0, each player is perturbed independently with probability $p$. If the perturbation occurs on a player $u$, we assume that the player $u$ chooses a new strategy uniformly at random from the interval $[0,10] \cap \Sigma$. Notice that for strategy $r_{u}>10$, the behavior of nodes is similar to $r_{u}=10$ as nodes only connect to the 4 grid neighbors. Let $\mathbf{r}^{0}$ be the strategy profile at time 0 after the perturbation. At each time step $t \geq 1$, every player picks the best strategy based on the strategies of others in the previous step: $r_{u}^{t}=\operatorname{argmax}_{r_{u} \in \Sigma \cap[0,10]} \pi\left(r_{u}, \mathbf{r}_{-u}^{t-1}\right), \forall u, \forall t>1$.

Figure 6 shows an extreme case where every player is perturbed when the initial profile is $\mathbf{r} \equiv 2$. The box-plot shows the distribution of players' strategies at each step. The figure shows that in just two steps the system returns to the navigable small-world NE. We tested 100 random starting profiles, and all of them converge to the navigable NE within two steps. This simulation result indicates that the navigable NE is very stable for random perturbations.

To contrast, we study the stability of the random small-world network in terms of tolerating perturbations. Figure 7 shows the result of randomly perturbing only $1 \%$ of players at the random NE, which are shown as the outliers at step 0 . Note that $1 \%$ perturbation does not meet the requirement in Theorem 3.7. However, this small fraction of players would affect the decision of additional players in their vicinity, who can significantly improve the reciprocity by also linking in the vicinity (indicated by Theorem 3.5). The figure clearly shows that in a few steps, more and more players would change their strategies, and 

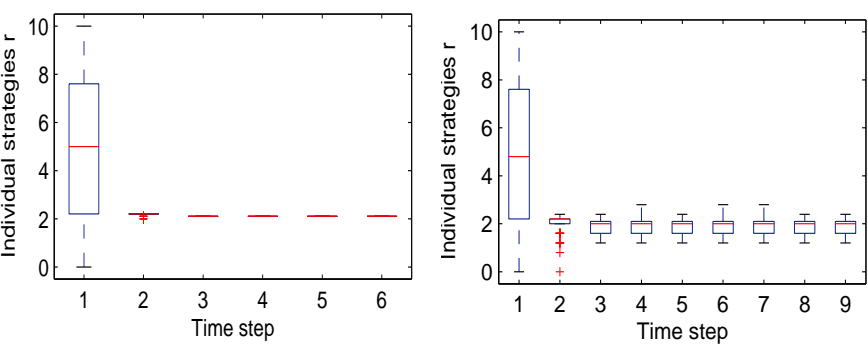

Fig. 8. Player only knows the Fig. 9. Player only knows the strategies of their friends (Noise strategies of their friends (Noise $\varepsilon=0)$. $\varepsilon \sim N(0,0.5))$

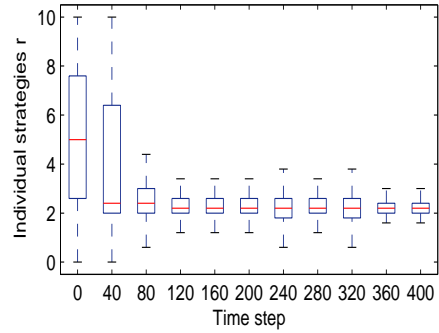

Fig. 10. Players have no knowledge of strategies of others.

the system finally goes to the navigable small-world NE. ${ }^{2}$ We tested 100 random starting profiles, and all of them converge to the navigable NE within at most 12 steps.

These results show that the navigable small-world NE are robust to perturbations, while random small-world NE is not stable and easily transits to the small-world NE under a slight perturbation.

\subsection{DRB Game with Limited Knowledge}

In practice, a player does not know the strategies of all players. So we now consider how to operate best response dynamics in practical scenarios. We first examine a weaker scenario where a player only knows the strategies of their friends. With these limited knowledge, a player can guess the strategies of all other players and pick the best response to the estimated strategies of all players. We next consider the weakest scenario where each player has no knowledge about the strategies of other players, and the only information needed is the empirical payoff observed by the player. To get this information, a player can create a certain number of links with the current strategy, and compute the payoff by multiplying the average link distance and the percentage of reciprocal links. In this scenario, players cannot directly calculate the best responses. Instead, they perform a heuristic search through choosing a response of better payoff than their current strategies, whenever they have opportunities to adjust the strategies. So as the time goes on, the player could change the strategy towards the best response.

Scenario 1: knowing friends' strategies. To examine the convergence of navigable small-world NE in this scenario, we simulate the DRB game as follows. At time step 0, each player chooses an initial strategy uniformly at random from the interval $[0,10] \cap \Sigma$. At every step $t \geq 0$, each player $u$ creates $q$ out-going long-range links based on her current strategy $r_{u}^{t}$, and learns the connection preferences of these $q$ long-range contacts. Let $F_{u}^{t}$ be the set of these $q$ long-range contacts. We further allows a random noise term $\varepsilon$ for each connection preference learned from the friends. Let $\hat{r}_{v}^{t}\left(v \in F_{u}^{t}\right)$ be the learned (noisy) connection preference. Then based on these newly learned connection preferences, player $u$ estimates the strategies of all other players. One reasonable estimation method is to assume that players close to one another in grid distance have similar strategy. More specifically, for a non-friend node $v \notin F_{u}^{t}, u$ estimates the strategy of $v$ by the average weight of known strategies: $\hat{r}_{v}^{t}=\frac{\sum_{f \in F_{u}^{t}} \hat{r}_{f, t-1} / d_{M}(v, f)}{\sum_{f \in F_{u}^{t}} 1 / d_{M}(v, f)}$.

Here we do not use the connection preferences learned in the previous steps and effectively assume that those old links are removed. This is both for convenience, and also reasonable

${ }^{2}$ In step 1 and 2 in Figure 7, the number of outliers is larger than in step 0, even though the rendering make it seems they are less. 


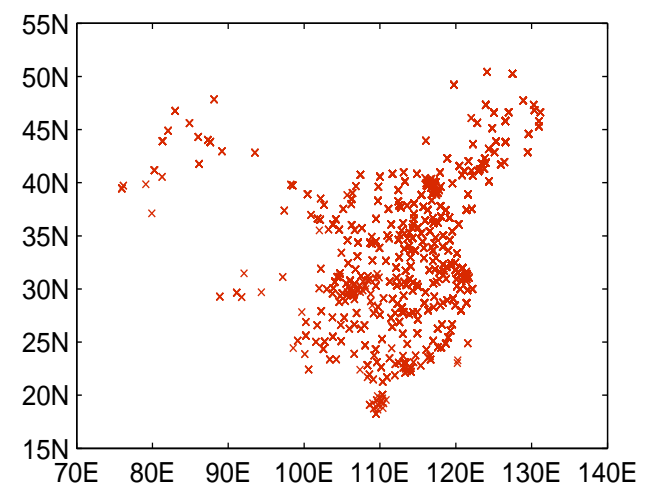

Fig. 11. The hometown location of Renren users.

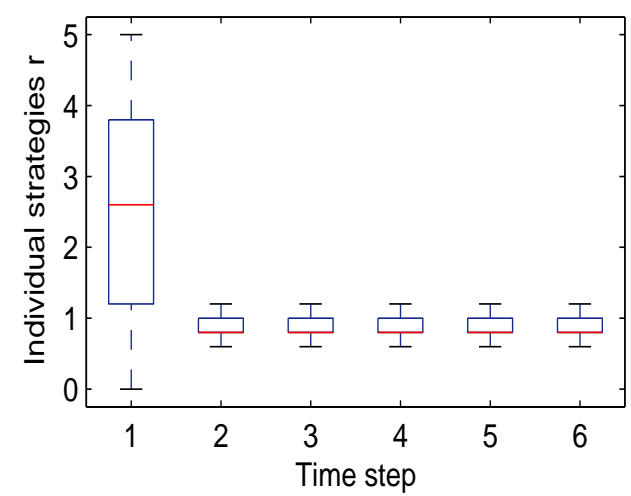

Fig. 12. Simulated network evolution over Renren grid.

since people could only maintain a limited number of connections and it is natural that new connections replace the old ones. Moreover, the connection preferences of those old connections may become out-dated in practice anyway. After the estimation procedure, player $u$ uses the strategy $\hat{r}_{v}^{t}$ from all other players (either learned or estimated) to compute its best response $\hat{r}_{u}^{t+1}$ for the next step.

In our experiment, we set $q=30$. Figure 8 shows that when players have accurate knowledge of the strategies of their friends without noise, the system converges in just two steps. Even when the information on friends' strategies is noisy, the system can still quickly stabilize in a few steps to a state close to the navigable small-world NE, as shown in Figure 9. We tested 100 random starting profiles and also other estimation methods such as randomly choosing a connection preference based on friends' connection preference distributions, and results are all similar. This experiment further demonstrates the robustness of the smallworld NE even under limited information on connection preferences.

Scenario 2: No information about others' strategies. To make it even harder, we do not allow the player to try many different strategies at each step before fixing her strategy for the step. Instead, at each step each player only has one chance to slightly modify her current strategy. If the new strategy yields better payoff, the player would adopt the new strategy. So as the time goes on, the player could change the strategy towards the best one.

We simulate the DRB game as follows: At time step 0, each player chooses an initial strategy uniformly at random from the interval $[0,10] \cap \Sigma$. Every player creates $q$ out-going links with her current strategy. At each time step $t \geq 1$, each player changes the strategy, i.e., $r_{u} \leftarrow r_{u}+\delta$, and creates $q$ new links with this new strategy, where $\delta$ is a random number determined as follows. First, for the sign of $\delta$, in the first step it is randomly assigned positive or negative sign with equal probability; in the remaining steps, to make the search efficient, we keep the sign of $\delta$ if the previous change leads to a higher payoff; otherwise we reverse the sign of $\delta$. For the magnitude of $\delta$, i.e. $|\delta|$, we sample a value uniformly at random from $(0,1] \cap \Sigma$.

We simulate this system with $q=30$. Figure 10 demonstrates that the system can still evolve to a state close to the navigable small-world NE in a few hundred steps, e.g., the strategies of $80.5 \%$ players fall in the interval $[1.8,2.4]$, and the median of the strategies is the navigable NE strategy of 2.1. We test 50 random starting profiles, and take snapshots of the strategy profiles at the time step $t=500$. On average, the strategies of $79.8 \%$ players in the snapshots fall in the interval $[1.8,2.4]$. 

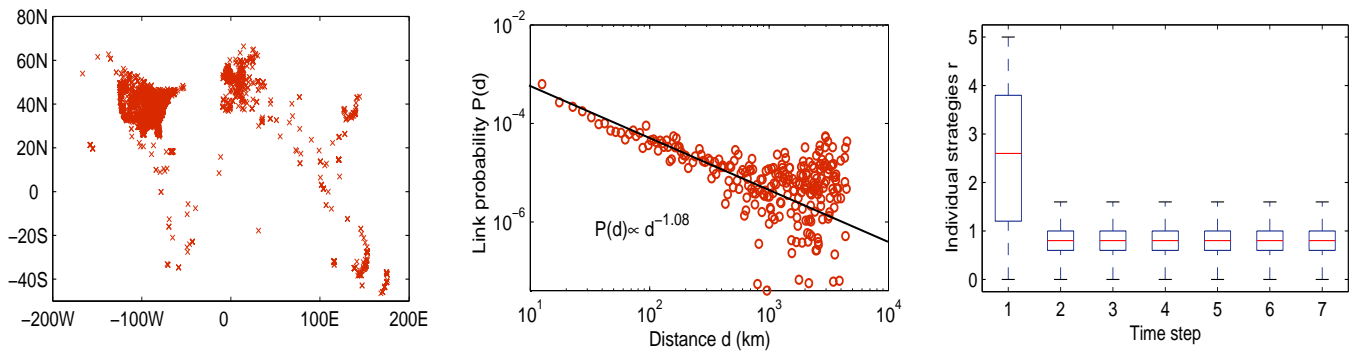

Fig. 13. The hometown location of F

Fig. 14. Friendship probability LiveJournal users. vs. distance in LiveJournal.

Fig. 15. Simulated network evolution over LiveJournal network.

In summary, our empirical evaluation strongly supports that our payoff function considering the balance between link distance and reciprocity naturally gives rise to the navigable small-world network. The convergence to navigable equilibrium will happen either when the players know all other players' strategies, or only learn their friends' strategies, or only use the empirical distance and reciprocity measure. Once in the navigable equilibrium, the system is very stable and hard to deviate by any random perturbation. Furthermore, other equilibria such as the random small world is not stable, in that a small perturbation will drive the system back to the navigable small-world network.

\subsection{DRB Game under Real Population Distribution}

Recall that real population is not evenly distributed geographically as in the Kleinberg's model. So we want to examine if our game could lead to an overall connection preference $r$ similar to the empirical one in the real network. To do so, we examine our game with the non-uniform geographic distribution of people in the following two real networks. We also introduce heterogeneity in players' tradeoff functions with $\alpha_{u}$ taken from a uniform distribution on $[0.1,10]$.

Renren Network. We sample $10 \mathrm{~K}$ users at random from Renren network, and we construct a real grid through mapping the hometown listed in users' profiles to (longitude, latitude) coordinates, as shown in Figure 11. To examine the convergence of navigable small-world NE in this scenario, we simulate the DRB game as follows. At time step 0, each player chooses an initial strategy uniformly at random from the interval $[0,5] \cap \Sigma$. At each time step $t \geq 1$, every player picks the best strategy based on the strategies of others in the previous step: $r_{u}^{t}=\operatorname{argmax}_{r_{u} \in \Sigma \cap[0,5]} \pi\left(r_{u}, \mathbf{r}_{-u}^{t-1}\right), \forall u, \forall t>1$.

Figure 12 shows that in a few steps, the system reaches a NE, where individual users adopt their respective equilibrium strategies. In the NE, the mean of the strategies is 0.85 and the strategies of $88.1 \%$ users fall in the interval $[0.7,1.1]$. So the overall connection preference of users in the simulated game is very close to the empirical value of 0.9 shown in Figure 2.

LiveJournal Network. To evaluate across-dataset generalization, we also examine our DRB game in the LiveJournal social network. LiveJournal is a community of bloggers with over 39 million registered users worldwide as the end of 2012. Each user provides a personal profile, including home location, personal interests and a list of other bloggers considered as friends. We crawl the profiles of 527,769 LiveJournal users used in the study [Liben-Nowell et al. 2005]. Given the 224,155 users providing city information, we successfully obtained a meaningful geographic location for only 197,504 users, as shown in Figure 13. To get the empirical connection preference of these LiveJournal users, we compute the friendship probability $p(d)$ for any given distance $d$ by the proportion of friendships among all pairs $(u, v)$ with distance $d$. Figure 14 shows the relationship between friendship 
probability and geographic distance, which shows that the real connection preference of users is around 1.08. These results demonstrates that our game does generalize across online social networks.

\section{DISCUSSION AND FUTURE WORK}

Our paper is a contribution to the literature on navigability and also on network formation games. There exists a plethora of results relating to network formation games in economics, as well as in computer science [Jackson 2004]. Existing games typically use pure-link-based strategy, and it is difficult for an individual to estimate the potential likelihood of forming reciprocal links to other users (i.e., the introduced notion of reciprocity). These games lead to mostly trivial equilibria such as cliques or stars. Different from prior games, our game uses the link probability functions as the strategies, and an individual is able to estimate reciprocity by learning the connection preferences of others. This difference in modeling methodology is substantive since it gives rise to the non-trivial structure of navigable small world networks. Also, most network formation games examine the strategically stable networks in static and non-perturbed settings. By contrast, our game examines the stability of equilibrium networks under the best response dynamics with the perturbation introduced. Dynamics could help select among different equilibria of the static game, the results in this paper illustrate this potential very well.

In the paper, we use a $k$-dimensional grid $(k \in \mathbb{N} \backslash\{0\})$ to be consistent with Kleinberg's small-world model, where each grid location contains a single node (a total of $n^{k}$ nodes). Let $B_{l}(u)$ denote the number of nodes within distance $l(l>0)$ from a node $u$. In fact, the key spatial property required in our analysis is $B_{l}(u)=\Theta\left(l^{D}\right), \forall u \in V, \forall D>0$, where $D$ is actually the fractional dimension proposed by Liben-Nowell et al. [Liben-Nowell et al. 2005]. So most of our results can be easily extended to a more general space that can be described the fractional dimension, where the grid is only the special case of integer dimension. The results in Section 5.3 actually provided empirical demonstration on Renren and LiveJournal latitude-longitude space that have a fractional dimension close to one. Similarly, we can also allow multiple nodes to be in the same location as long as the spatial property of $B_{l}(u)=\Theta\left(l^{D}\right), \forall u \in V$ holds for $l>0$, i.e., the space could still be described by the fractional dimension. Given $p=0$ in our setting $K(n, k, p, q, \mathbf{r})$, each node has undirected edges connecting to all other nodes in the same location as local contacts. As we have discussed in Section 2.3, we do not consider these local contacts in our game.

The population size and the payoff obtained at the critical value are sufficiently large to allow us to ignore stochastic effects. In our game, the environment state consists of (i) the geographical distribution of users, which remains stable over time given the large population size; and (ii) the tradeoff factor $\alpha_{u}\left(\alpha_{u}>0\right)$ for any user $u$, which has no influence on the strategy choice over time, as implied by lemma 3.1. Specifically, a node $u$ chooses $r_{u}=k$ once it has a nearby node $w$ satisfying $r_{w} \geq k$ or $r_{w}=\max _{v V} r_{v}$, irrespective of the tradeoff factor every node chooses (including the $u$ itself).

Our study opens many possible directions of future work. For example, one may provide a theoretical analysis of the DRB game on the non-uniform population distributions, which has been empirically validated by our experiments on the Renren and LiveJournal datasets. Another direction is to integrate prior studies on human mobility model to provide a more complete picture of the underlying mechanisms for navigable small-world networks. For example, one could use move-and-forget mobility model [Chaintreau et al. 2008] to generate link probability functions of power law form, and adopt our game-theoretic approach to drive individuals to choose the critical one enabling navigability to arise. It is also interesting to investigate the existence of other forms of utility functions, since given the complex human behavior in the real world, there might be more behavioral factors leading to the actual real small world. We wish our study could encourage more empirical and theoretical studies on 
the relationship between reciprocity, distance, and navigability, and perhaps uncover the underlying human behavior model that integrates these factors together.

\section{REFERENCES}

Lada Adamic and Eytan Adar. 2005. How to search a social network. Social Networks 27 (2005).

K Bhattacharya, G Mukherjee, J Saramäki, K Kaski, and S S Manna. 2008. The International Trade Network: weighted network analysis and modelling. JSTAT (2008).

M. Boguñá, D. Krioukov, and K.C. Claffy. 2009. Navigability of complex networks. Nature Physics 5 (2009), $74-80$.

Augustin Chaintreau, Pierre Fraigniaud, and Emmanuelle Lebhar. 2008. Networks Become Navigable as Nodes Move and Forget. In Proc. of ICALP '08.

Wei Chen, Wenjie Fang, Guangda Hu, and Michael W. Mahoney. 2013. On the Hyperbolicity of Small-World and Treelike Random Graphs. Internet Mathematics 9, 4 (2013), 434-491.

Eunjoon Cho, Seth A. Myers, and Jure Leskovec. 2011. Friendship and Mobility: User Movement In LocationBased Social Networks. In Proc. of KDD.

Aaron Clauset and Cristopher Moore. 2003. How Do Networks Become Navigable. In Arxiv preprint arXiv:0309.415v2.

Ross Gittell and Avis Vidal. 1998. Community Organizing: Building Social Capital as a Development Strategy. Sage publicaiton.

Jacob Goldenberg and Moshe Levy. 2009. Distance Is Not Dead: Social Interaction and Geographical Distance in the Internet Era. In Arxiv preprint arXiv:0906.3202.

Marta C. Gonzlez, Csar A. Hidalgo, and Albert-Lszl Barabsi. 2008. Understanding individual human mobility patterns. nature (2008).

Alvin Ward Gouldner. 1960. The norm of reciprocity: a preliminary statement. American Sociological Review 25, 4 (1960), 161-178.

Mark Granovetter. 1974. Getting a job: A study of contacts and careers. Harvard University Press.

Mark S Granovetter. 1973. The strength of weak ties. Amer. J. Sociology (1973), 1360-1380.

András Gulyás, József J. Bíró, Attila Korösi, Gábor Rétvári, and Dmitri Krioukov. 2015. Navigable Networks as Nash Equilibria of Navigation Games. Nature Communications 6 (2015), 7651.

András Gulyás, Attila Korösi, Dávid Szabó, and Gergely Biczóki. 2012. On greedy network formation. SIGMETRICS Perform. Eval. Rev. (2012).

Yanqing Hu, Yougui Wang, Daqing Li, Shlomo Havlin, , and Zengru Di. 2011. Possible Origin of Efficient Navigation in Small Worlds. Phys Rev Letters (2011).

Johannes Illenberger, Kai Nagel, and Gunnar Flötteröd. 2013. The Role of Spatial Interaction in Social Networks. Networks and Spatial Economics 13 (2013).

Matthew O. Jackson. 2004. A Survey of Models of Network Formation: Stability and Eciency. Group Formation in Economics: Networks, Clubs and Coalitions (2004).

Akshay Java, Xiaodan Song, Tim Finin, and Belle Tseng. 2007. Why we twitter: understanding microblogging usage and communities. In Proc. of WebKDD/SNA-KDD.

J. Jiang, C. Wilson, X. Wang, P. Huang, W. Sha, Y. Dai, and B. Y. Zhao. 2010. Understanding Latent Interactions in Online Social Networks. In Proc. of IMC.

Jon Kleinberg. 2002. The Small-World Phenomenon: An Algorithmic Perspective. In Proc. of 32nd ACM Symp. on Theory of Computing (STOC).

Jon Kleinberg. 2006. Complex networks and decentralized search algorithms. In Proc. of the International Congress of Mathematicians (ICM).

Gautier Krings, Francesco Calabrese, Carlo Ratti, and Vincent D Blondel. 2009. Urban Gravity: a Model for Intercity Telecommunication Flows. J. Stat. Mech.-Theor. Exp. (2009).

D. Krioukov, F. Papadopoulos, M. Kitsak, A. Vahdat, and M. Boguñá. 2010. Hyperbolic Geometry of Complex Networks. Physical Review E 82 (2010), 036106.

D. Krioukov, F. Papadopoulos, A. Vahdat, and M. Boguñá. 2009. Curvature and temperature of complex networks. Physical Review E 80 (2009), 035101(R).

Renaud Lambiotte and others. 2008. Geographical dispersal of mobile communication networks. Physica A 387 (2008).

David Liben-Nowell, Jasmine Novak, Ravi Kumar, Prabhakar Raghavan, and Andrew Tomkins. 2005. Geographic routing in social networks. PNAS 102 (2005).

Nisha Mathias and Venkatesh Gopal. 2001. Small worlds: how and why. Physical Review E 63, 2 (2001). 
Stanley Milgram. 1967. The small world problem. Psychology Today 2, 1 (1967), 60-67.

Alan Mislove, Massimiliano Marcon, Krishna P. Gummadi, Peter Druschel, and Bobby Bhattacharjee. 2007. Measurement and analysis of online social networks. In Proc. of IMC.

Fragkiskos Papadopoulos, Dmitri V. Krioukov, Marián Boguñá, and Amin Vahdat. 2010. Greedy Forwarding in Dynamic Scale-Free Networks Embedded in Hyperbolic Metric Spaces. In Proc. of INFOCOM.

Oskar Sandberg and Ian Clarke. 2006. The Evolution of Navigable Small-World Networks. In Arxiv preprint arXiv:cs/0607025.

Mark Schaller and Bibb Latank. 1995. Distance matters: Physical space and social impact. Personality and Social Psychology Bulletin 25 (1995).

Éva Tardos and Tom Wexler. 2007. Network formation games and the potential function method. (2007). In Algorithmic Game Theory.

Duncan J Watts and Steven H Strogatz. 1998. Collective dynamics of 'small-world' networks. Nature 393, 6684 (1998), 440-442.

Zhi Yang, Christo Wilson, Xiao Wang, Tingting Gao, Ben Y. Zhao, and Yafei Dai. 2011. Uncovering Social Network Sybils in the Wild. In Proc. of IMC.

Kai Zhao, Mirco Musolesi, Pan Hui, Weixiong Rao, and Sasu Tarkoma. 2015. Explaining the power-law distribution of human mobility through transportation modality decomposition. Scientific Reports (2015). 
ta-

tion

\begin{tabular}{|c|c|}
\hline$k, n$ & Dimension and edge length of a grid $\underbrace{n \times n \times \ldots \times n}_{k}$ \\
\hline$n_{D}$ & Diameter of grid, $n_{D}=k\lfloor n / 2]$ \\
\hline$V$ & Set of players \\
\hline$p, q$ & Number of local and long-range contacts \\
\hline$d_{M}(u, v)$ & Manhattan distance between players $u$ and $v$ \\
\hline$r_{u}$ & Connection preference of player $u$ \\
\hline$\alpha_{u}$ & Constant exponent for player $u$ 's distance-reciprocity tradeoff, $\left(\alpha_{u}>0\right)$ \\
\hline$c\left(r_{u}\right)$ & Normalization constant $c\left(r_{u}\right)=\sum_{\forall v \neq u} d_{M}(u, v)^{-r u}$ \\
\hline$p_{u}\left(v, r_{u}\right)$ & Probability that $u$ connects $v$ under $r_{u}, p_{u}\left(v, r_{u}\right)=d_{M}(u, v)^{-r_{u}} / c\left(r_{u}\right)$ \\
\hline $\mathbf{r}$ & Vector of $r_{u}$ values on all players (strategy profile) \\
\hline$\pi_{u}\left(r_{u}, \mathbf{r}_{-\mathbf{u}}\right)$ & Player $u$ 's payoff given the strategy profile $\mathbf{r}$ \\
\hline$D\left(r_{u}\right)$ & Average link distance of $u, D\left(r_{u}\right)=\sum_{\forall v \neq u} p_{u}\left(v, r_{u}\right) d_{M}(u, v)$ \\
\hline$P_{u}\left(r_{u}, \mathbf{r}_{-u}\right)$ & Reciprocity of $u, P_{u}\left(r_{u}, \mathbf{r}_{-u}\right)=\sum_{\forall v \neq u} p_{u}\left(v, r_{u}\right) p_{v}\left(u, r_{v}\right)$ \\
\hline$\gamma$ & Granularity of connection preference, strategy set $\Sigma=\{0, \gamma, 2 \gamma, 3 \gamma, \ldots\}$, \\
\hline$b_{u}(j)$ & The number of players at grid distance $j$ from $u$ \\
\hline$\xi_{k}^{-}$ & Constant making $b_{u}(j) \geq \xi_{k}^{-} j^{k-1}$ for $1 \leq j \leq\lfloor n / 2\rfloor$ \\
\hline$\xi_{k}^{+}$ & Constant making $b_{u}(j) \leq \xi_{k}^{+} j^{k-1}$ for $j \leq n_{D}$ \\
\hline$\varepsilon$ & Deviation from strategy $k, \varepsilon=k-r_{u}$ \\
\hline
\end{tabular}

\section{A. COMMONLY USED RESULTS ON THE KLEINBERG'S SMALL WORLD AND THE DRB GAME}

In all proofs in the appendix, for a given node $u \in V$, we denote $D\left(r_{u}\right)=$ $\sum_{\forall v \neq u} p_{u}\left(v, r_{u}\right) d_{M}(u, v)$ as its average grid distance of its long range contacts (simply referred to as the link distance), and $P_{u}\left(r_{u}, \mathbf{r}_{-u}\right)=\sum_{\forall v \neq u} p_{u}\left(v, r_{u}\right) p_{v}\left(u, r_{v}\right)$ as its reciprocity. When $\mathbf{r}_{-u} \equiv s$, we simply use $P\left(r_{u}, s\right)$ to denote $P_{u}\left(r_{u}, \mathbf{r}_{-u} \equiv s\right)$. Moreover, for any $A \subseteq V$, let $P_{u, A}(\mathbf{r})=\sum_{v \in A} p_{u}\left(v, r_{u}\right) p_{v}\left(u, r_{v}\right)$ be the reciprocity $u$ obtained from subset $A$. We denote $c\left(r_{u}\right)=\sum_{\forall v \neq u} d_{M}(u, v)^{-r_{u}}$ as $u$ 's normalized coefficient. The subscript $u$ in $D\left(r_{u}\right), P\left(r_{u}, s\right)$ and $c\left(r_{u}\right)$ is omitted because their values are the same for all $u \in V$.

Let $n_{D}$ be the longest grid distance among nodes in $K(n, k, p, q, \mathbf{r})$. We have that $n_{D}=$ $k\lfloor n / 2\rfloor$. We denote $b_{u}(j)$ as the number of players at grid distance $j$ from $u$. We can find two constants $\xi_{k}^{-}$and $\xi_{k}^{+}$only depending on the dimension $k$, so that $\xi_{k}^{-} j^{k-1} \leq b_{u}(j) \leq \xi_{k}^{+} j^{k-1}$ for $1 \leq j \leq\lfloor n / 2\rfloor$ and $1 \leq b_{u}(j) \leq \xi_{k}^{+} j^{k-1}$ for $\lfloor n / 2\rfloor<j \leq n_{D} \cdot{ }^{3}$ Note that the payoff function for the DRB game is indifferent of parameters $p$ and $q$ of the network, so we treat $p=q=1$ for our convenience in the analysis.

Recall that we assume that each $r_{u}$ is taken from a discrete set $\Sigma=\{0, \gamma, 2 \gamma, 3 \gamma, \ldots$,$\} ,$ where $\gamma$ represents the granularity of connection preference and is in the form of $1 / g$ for some positive integer $g \geq 2$. Using discrete strategy set avoids nuances in continuous strategy space and is also reasonable in practice since people are unlikely to make infinitesimal changes. Henceforth, for any $r_{u} \neq k$, we have $\left|k-r_{u}\right| \geq \gamma$. The notation commonly used in the paper is described in Table I.

We first show the following two lemmas, which will be used in the most of theorems.

${ }^{3}$ The exact values of $\xi_{k}^{-}$and $\xi_{k}^{+}$can be derived by the combinatorial problem of counting the number of ways to choose $k$ non-negative integers such that they sum to a given positive integer $j$. 
Lemma A.1. In the $k$-dimensional grid $K(n, k, p, q, \mathbf{r})$, for a given node $u \in V$ with a strategy of $r_{u}$, the normalized coefficient $c\left(r_{u}\right)$ has the following bounds:

$$
\begin{cases}\frac{\xi_{k}^{-}}{2^{k+1} k} n^{k-r_{u}} \leq c\left(r_{u}\right) \leq \xi_{k}^{+} k^{k-r_{u}} n^{k-r_{u}} & \text { if } r_{u}<k, \\ \frac{\xi_{k}^{-} \ln n}{2} \leq c\left(r_{u}\right) \leq \xi_{k}^{+} \ln (2 k n) & \text { if } r_{u}=k, \\ \xi_{k}^{-} \leq c\left(r_{u}\right) \leq \xi_{k}^{+}(1+1 / \gamma) & \text { if } r_{u}>k .\end{cases}
$$

Proof. In the case of $r_{u}<k$, we write $\varepsilon=k-r_{u}(\gamma \leq \varepsilon \leq k)$. The coefficient $c\left(r_{u}\right)$ can be bounded as:

$$
\begin{aligned}
c\left(r_{u}\right)= & \sum_{\forall v \neq u} d_{M}(u, v)^{-r_{u}} \geq \sum_{j=1}^{n / 2} b_{u}(j) j^{-r_{u}} \geq \xi_{k}^{-} \sum_{j=1}^{n / 2} j^{\varepsilon-1} \\
& \geq \xi_{k}^{-} \int_{1}^{n / 2} x^{\varepsilon-1} d x \geq \frac{\xi_{k}^{-}}{\varepsilon}\left(\frac{n}{2}\right)^{\varepsilon}-\frac{\xi_{k}^{-}}{\varepsilon} \geq \frac{\xi_{k}^{-}}{2 \varepsilon}\left(\frac{n}{2}\right)^{\varepsilon}
\end{aligned}
$$

The last inequality above relies on a loose relaxation of $\frac{1}{2}\left(\frac{n}{2}\right)^{\varepsilon} \geq 1$, which is guaranteed for all $n \geq 2^{1+1 / \gamma}$ since $\varepsilon \geq \gamma$. Note that $\varepsilon<k$, so we have:

$$
c\left(r_{u}\right) \geq \frac{\xi_{k}^{-}}{2^{1+\varepsilon} \varepsilon} n^{\varepsilon} \geq \frac{\xi_{k}^{-}}{2^{1+k} k} n^{\varepsilon} .
$$

The upper bound of coefficient $c\left(r_{u}\right)$ can be given as:

$$
\begin{aligned}
& c\left(r_{u}\right)=\sum_{\forall v \neq u} d_{M}(u, v)^{-r_{u}}=\sum_{j=1}^{n_{D}} b_{u}(j) j^{-r_{u}} \leq \xi_{k}^{+} \sum_{j=1}^{n_{D}} j^{\varepsilon-1} \\
& \leq \begin{cases}1+\xi_{k}^{+} \int_{1}^{n_{D}} j^{\varepsilon-1} d x \leq 1+(k n / 2)^{\varepsilon} & \text { if } \varepsilon<1, \\
\xi_{k}^{+} \int_{j=1}^{n_{D}+1} j^{\varepsilon-1} d x \leq \xi_{k}^{+}(k n / 2+1)^{\varepsilon} & \text { if } \varepsilon \geq 1,\end{cases} \\
& \leq \xi_{k}^{+} k^{\varepsilon} n^{\varepsilon} \text {. }
\end{aligned}
$$

The last inequality above relies on a loose relaxation of $\frac{k n}{2} \geq 1$, which is guaranteed for all $n \geq 2$ since $k \geq 1$.

We now turn to the case of $r_{u}=k$. The upper bound of normalization coefficient $c(k)$ can be given as

$$
c(k)=\sum_{\forall v \neq u} d_{M}(u, v)^{-k}=\sum_{j=1}^{n_{D}} b_{u}(j) j^{-k} \leq \xi_{k}^{+} \sum_{j=1}^{n_{D}} \frac{1}{j} \leq \xi_{k}^{+} \ln (2 k n),
$$

and its lower bound is

$$
c(k) \geq \xi_{k}^{-} \sum_{j=1}^{n / 2} j^{-1} \geq \xi_{k}^{-} \int_{1}^{n / 2} x^{-1} d x \geq \xi_{k}^{-}(\ln n-\ln 2) \geq \frac{\xi_{k}^{-} \ln n}{2} .
$$

where the last inequality is true when $n \geq e^{4}$.

We finally consider the the case of $r_{u}>k$, it is easy to get that

$$
c\left(r_{u}\right)=\sum_{\forall v \neq u} d_{M}(u, v)^{-r_{u}} \geq \sum_{j=1}^{n / 2} b_{u}(j) j^{-r} \geq b_{u}(1) \geq \xi_{k}^{-},
$$

and its upper bound is given as:

$$
c\left(r_{u}\right)=\sum_{\forall v \neq u} d_{M}(u, v)^{-r_{u}}=\sum_{j=1}^{n_{D}} b_{u}(j) j^{-r_{u}} \leq \xi_{k}^{+} \sum_{j=1}^{n_{D}} j^{k-1} j^{-r_{u}} \leq \xi_{k}^{+} \sum_{j=1}^{n_{D}} j^{-\left(r_{u}-k\right)-1} .
$$


Note $r_{u}-k \geq \gamma$, we have:

$$
c\left(r_{u}\right) \leq \xi_{k}^{+} \sum_{j=1}^{n_{D}} j^{-\left(r_{u}-k\right)-1} \leq \xi_{k}^{+} \sum_{j=1}^{n_{D}} j^{-\gamma-1} \leq \xi_{k}^{+}\left(1+\int_{1}^{n_{D}+1} x^{-\gamma-1} d x\right) \leq \xi_{k}^{+}\left(1+\frac{1}{\gamma}\right) .
$$

Lemma A.2. In the $k$-dimensional grid $K(n, k, p, q, \mathbf{r})$, for a given node $u \in V$ with a strategy of $r_{u}$, the average distance of its long-range contacts $D\left(r_{u}\right)$ has the following bounds:

$$
\left\{\begin{aligned}
D\left(r_{u}\right) \leq \frac{\xi_{k}^{+} k^{1+k}}{c\left(r_{u}\right)} n^{1+k-r_{u}} & & \text { if } r_{u}<k, \\
\frac{\xi_{k}^{-} n}{2 c(k)} \leq D\left(r_{u}\right) \leq \frac{\xi_{k}^{+} n}{c(k)} & & \text { if } r_{u}=k, \\
D\left(r_{u}\right) \leq \frac{\xi_{k}^{+} k}{2 \gamma c\left(r_{u}\right)} n^{1-\gamma} & & \text { if } k<r_{u}<k+1, \\
D\left(r_{u}\right) \leq \frac{\xi_{k}^{+}}{c\left(r_{u}\right)} \ln (2 k n) . & & \text { if } r_{u} \geq k+1 .
\end{aligned}\right.
$$

Proof. When $r_{u}<k$, we write $\varepsilon=k-r_{u}(\gamma \leq \varepsilon \leq k)$ and get the upper bound for the link distance

$$
D\left(r_{u}\right)=\frac{\sum_{j=1}^{n_{D}} b_{u}(j) \cdot j^{-r_{u}} \cdot j}{c\left(r_{u}\right)} \leq \frac{\xi_{k}^{+} \int_{1}^{n_{D}+1} x^{\varepsilon} d x}{c\left(r_{u}\right)} \leq \frac{\xi_{k}^{+}\left(n_{D}+1\right)^{1+\varepsilon}}{(1+\varepsilon) c\left(r_{u}\right)} \leq \frac{\xi_{k}^{+}(k n)^{1+\varepsilon}}{c\left(r_{u}\right)} \leq \frac{\xi_{k}^{+} k^{1+k}}{c\left(r_{u}\right)} n^{1+\varepsilon} .
$$

We now turn to the case of $r_{u}=k$. The upper bound of link distance $D(k)$ can be given as

$$
D\left(r_{u}=k\right)=\frac{\sum_{j=1}^{n_{D}} b_{u}(j) \cdot j^{-k} \cdot j}{c\left(r_{u}\right)} \leq \frac{\xi_{k}^{+} n}{c(k)}
$$

and its lower bound is

$$
D\left(r_{u}\right) \geq \frac{\sum_{j=1}^{n / 2} b_{u}(j) \cdot j^{-k} \cdot j}{c(k)} \geq \frac{\xi_{k}^{-} n}{2 c(k)}
$$

We finally consider the case of $r_{u}>k$. We write $\varepsilon=r_{u}-k(\varepsilon \geq \gamma)$, and the bound for the link distance is:

$$
D\left(r_{u}\right)=\frac{\sum_{j=1}^{n_{D}} b_{u}(j) \cdot j^{-r_{u}} \cdot j}{c\left(r_{u}\right)} \leq \xi_{k}^{+} \sum_{j=1}^{n_{D}} \frac{j^{-\varepsilon}}{c\left(r_{u}\right)} \leq \xi_{k}^{+} \frac{1+\int_{1}^{n_{D}} x^{-\varepsilon} d x}{c\left(r_{u}\right)}
$$

In the case of $\varepsilon<1$,

$$
D\left(r_{u}\right) \leq \xi_{k}^{+} \frac{1+\int_{1}^{n_{D}} x^{-\varepsilon} d x}{c\left(r_{u}\right)} \leq \frac{\xi_{k}^{+}}{(1-\varepsilon) c\left(r_{u}\right)}(k n / 2)^{1-\varepsilon} \leq \frac{\xi_{k}^{+} k}{2 \gamma c\left(r_{u}\right)} n^{1-\varepsilon} \leq \frac{\xi_{k}^{+} k}{2 \gamma c\left(r_{u}\right)} n^{1-\gamma} .
$$

otherwise,

$$
D\left(r_{u}\right) \leq \xi_{k}^{+} \frac{1+\int_{1}^{n_{D}} x^{-\varepsilon} d x}{c\left(r_{u}\right)} \leq \frac{\xi_{k}^{+}}{c\left(r_{u}\right)} \ln (2 k n)
$$

Lemma A.3. In the $k$-dimensional DRB game, there exists a constant $\kappa$ (only depending on model constants $k$ and $\gamma$ ), for sufficiently large $n$ (in particular $n \geq \max \left(e^{4}, 2 k\right)$ ), the 
following statement holds: for any strategy profile $\mathbf{r}$, any node $u$ with $r_{u} \neq k$ and $\alpha_{u}>0$, $\pi_{u}\left(r_{u}, \mathbf{r}_{-u}\right) \leq \kappa n^{\alpha_{u}-\min \left\{1, \alpha_{u}\right\} \gamma}$.

Proof. We introduce some notations first. Given the strategy profile $\mathbf{r}$ and a node $u$ with $r_{u} \neq k$, we partition the rest nodes $V \backslash\{u\}$ into three sets: $V_{<k}=\left\{v \in V \backslash\{u\} \mid r_{v}<k\right\}$, $V_{>k}=\left\{v \in V \backslash\{u\} \mid r_{v}>k\right\}, V_{=k}=\left\{v \in V \backslash\{u\} \mid r_{v}=k\right\}$. Then we have

$$
\pi_{u}(\mathbf{r})=D\left(r_{u}\right)^{\alpha_{u}}\left(P_{u, V_{<k}}(\mathbf{r})+P_{u, V_{>k}}(\mathbf{r})+P_{u, V_{=k}}(\mathbf{r})\right) .
$$

We now consider the case of $r_{u}<k$ and $r_{u}>k$ separately.

Payoff of $r_{u}<k$. Let $\varepsilon=k-r_{u}(\gamma \leq \varepsilon \leq k)$. We first consider the average grid distance to long-range contacts in this case. Based on the bound on $D\left(r_{u}\right)$ and $c\left(r_{u}\right)$ given by inequalities (A.2a) and (A.1a), we get:

$$
D\left(r_{u}\right)^{\alpha_{u}}=\frac{\xi_{k}^{+} k^{1+k}}{c\left(r_{u}\right)} n^{1+\varepsilon}=\frac{\xi_{k}^{+} k^{1+k}}{\frac{\xi_{k}^{-}}{2^{k+1} k} n^{\varepsilon}} n^{1+\varepsilon}=\frac{2^{k+1} \xi_{k}^{+} k^{2+k}}{\xi_{k}^{-}} n
$$

We now examine the reciprocity. We first consider the reciprocity player $u$ obtains from the players in $V_{<k}$. We have $c\left(r_{v}\right) \geq c(k-\gamma)$ for $\forall v \in V_{<k}$, since $r_{v} \leq k-\gamma$. Then we have:

$$
P_{u, V_{<k}}(\mathbf{r})=\sum_{v \in V_{<k}} \frac{d_{M}(u, v)^{-r_{u}-r_{v}}}{c\left(r_{u}\right) c\left(r_{v}\right)} \leq \sum_{v \in V_{<k}} \frac{d_{M}(u, v)^{-r_{u}}}{c\left(r_{u}\right) c(k-\gamma)} \leq \frac{\sum_{\forall v \neq u} d_{M}(u, v)^{-r_{u}}}{c\left(r_{u}\right) c(k-\gamma)}=\frac{1}{c(k-\gamma)}
$$

Combining with the inequalities (A.4) and (A.1a), we get:

$$
D\left(r_{u}\right)^{\alpha_{u}} P_{u, V_{<k}}(\mathbf{r}) \leq\left(\frac{2^{k+1} \xi_{k}^{+} k^{2+k}}{\xi_{k}^{-}} n\right)^{\alpha_{u}} \frac{1}{\frac{\xi_{k}^{-}}{2^{k+1} k} n^{\gamma}} \leq \frac{\left(\xi_{k}^{+}\right)^{\alpha_{u}} 2^{(k+1)\left(\alpha_{u}+1\right)} k^{(k+2) \alpha_{u}+1}}{\left(\xi_{k}^{-}\right)^{\alpha_{u}+1}} n^{\alpha_{u}-\gamma} .
$$

Next we examine the reciprocity that player $u$ obtains from the players in $V_{>k}$. Note that for all $v \in V_{>k}, r_{v} \geq k+\gamma$. Using the bound on $c\left(r_{v}\right)$ given by inequality (A.1c), we have:

$$
\begin{aligned}
& P_{u, V_{>k}}(\mathbf{r})=\sum_{v \in V_{<k}} \frac{d_{M}(u, v)^{-r_{u}-r_{v}}}{c\left(r_{u}\right) c\left(r_{v}\right)} \leq \frac{\sum_{j=1}^{n_{D}} b_{u}(j) \cdot j^{-r_{u}} \cdot j^{-k-\gamma}}{\xi_{k}^{-} c\left(r_{u}\right)}=\frac{\xi_{k}^{+} \sum_{j=1}^{n_{D}} j^{-1-r_{u}-\gamma}}{\xi_{k}^{-} c\left(r_{u}\right)} \\
& \leq \frac{\xi_{k}^{+}\left(1+\int_{1}^{n_{D}} x^{-1-r_{u}-\gamma} d x\right)}{\xi_{k}^{-} c\left(r_{u}\right)} \leq \frac{\xi_{k}^{+}\left(1+r_{u}+\gamma\right)}{\xi_{k}^{-}\left(r_{u}+\gamma\right) c\left(r_{u}\right)} \leq \frac{\xi_{k}^{+}(k+1)}{\xi_{k}^{-} \gamma c\left(r_{u}\right)} .
\end{aligned}
$$

Based on the bound on $D\left(r_{u}\right)$ and $c\left(r_{u}\right)$ given by inequalities (A.4) and (A.1a), we get:

$$
\begin{aligned}
D\left(r_{u}\right)^{\alpha_{u}} P_{u, V_{>k}}(\mathbf{r}) & \leq\left(\frac{2^{k+1} \xi_{k}^{+} k^{2+k}}{\xi_{k}^{-}} n\right)^{\alpha_{u}} \cdot \frac{\xi_{k}^{+}(k+1)}{\xi_{k}^{-} \gamma \frac{\xi_{k}^{-}}{2^{k+1} k} n^{\varepsilon}} \\
& \leq \frac{\left(\xi_{k}^{+}\right)^{\alpha_{u}+1} 2^{(k+1)\left(\alpha_{u}+1\right)} k^{(k+2) \alpha_{u}+1}(k+1)}{\left(\xi_{k}^{-}\right)^{\alpha_{u}+2} \gamma} n^{\alpha_{u}-\varepsilon} \\
& \leq \frac{\left(\xi_{k}^{+}\right)^{\alpha_{u}+1} 2^{(k+1)\left(\alpha_{u}+1\right)+1} k^{(k+2) \alpha_{u}+2}}{\left(\xi_{k}^{-}\right)^{\alpha_{u}+2} \gamma} n^{\alpha_{u}-\gamma} .
\end{aligned}
$$

We now examine the payoff of player $u$ from players in $V_{=k}$. In this case, the upper bound for the reciprocity is:

$$
P\left(r_{u}, k\right)=\frac{\sum_{j=1}^{n_{D}} b_{u}(j) \cdot j^{-r_{u}} \cdot j^{-k}}{c\left(r_{u}\right) c(k)} \leq \xi_{k}^{+} \sum_{j=1}^{n_{D}} \frac{j^{\varepsilon-1-k}}{c\left(r_{u}\right) c(k)} .
$$


Notice that $\varepsilon \leq k$, we have:

$$
P\left(r_{u}, k\right) \leq \frac{\xi_{k}^{+}}{c\left(r_{u}\right) c(s)}\left(1+\int_{j=1}^{n_{D}} j^{\varepsilon-1-s}\right) \leq \begin{cases}\frac{\xi_{k}^{+}(1+k-\varepsilon)}{(k-\varepsilon) c(r u c) c(k)} \leq \frac{2 k \xi_{k}^{+}}{\gamma c\left(r_{u}\right) c(k)} & \text { if } \varepsilon<k, \\ \frac{2 \xi_{k}^{+} \ln (2 k n)}{c\left(r_{u}\right) c(k)} & \text { if } \varepsilon=k,\end{cases}
$$

The inequalities in the cases above use the facts $\gamma \leq \varepsilon \leq k$ and $k-\varepsilon \geq \gamma$ when $\varepsilon<k$.

Combining the the above bounds on reciprocity with bounds given by inequalities (A.4), (A.1a) and (A.1b), we have the payoff of node $u$ getting from $V_{=k}$ :

$$
\begin{aligned}
& D\left(r_{u}\right)^{\alpha_{u}} P_{u, V_{=k}}(\mathbf{r}) \leq D\left(r_{u}\right)^{\alpha_{u}} P\left(r_{u}, k\right) \\
& \leq \begin{cases}\left(\frac{2^{k+1} \xi_{k}^{+} k^{2+k}}{\xi_{k}^{-}} n\right)^{\alpha_{u}} \cdot \frac{2 k \xi_{k}^{+}}{\gamma \frac{\xi_{k}^{-} n^{\varepsilon}}{2^{k+1}} \cdot \frac{\xi_{k}^{-} \ln n}{2}} & \text { if } \varepsilon<k, \\
\left(\frac{2^{k+1} \xi_{k}^{+} k^{2+k}}{\xi_{k}^{-}} n\right)^{\alpha_{u}} \cdot \frac{2 \xi_{k}^{+} \ln (2 k n)}{\frac{\xi_{k}^{-} n^{\varepsilon}}{2^{k+1}} \cdot \frac{\xi_{k}^{-} \ln n}{2}} & \text { if } \varepsilon=k,\end{cases} \\
& \leq \begin{cases}\frac{\left(\xi_{k}^{+}\right)^{\alpha_{u}+1} 2^{\alpha_{u}(k+1)+k+3} k^{\alpha_{u}(k+2)+1}}{\gamma\left(\xi_{k}^{-}\right)^{\alpha_{u}+2}} \frac{n^{\alpha u-\gamma}}{\ln n} & \text { if } \varepsilon<k . \\
\frac{\left(\xi_{k}^{+}\right)^{\alpha_{u}+1} 2^{\alpha_{u}(k+1)+k+3} k^{\alpha_{u}(k+2)+1} \ln (2 k n)}{\left(\xi_{k}^{-}\right)^{\alpha_{u}+2} \ln n} n^{\alpha_{u}-\gamma} \leq \frac{\left(\xi_{k}^{+}\right)^{\alpha_{u}+1} 2^{\alpha_{u}(k+1)+k+4} k^{\alpha_{u}(k+2)+1}}{\left(\xi_{k}^{-}\right)^{\alpha_{u}+2}} n^{\alpha_{u}-\gamma} & \text { if } \varepsilon=k .\end{cases}
\end{aligned}
$$

The last inequality in the case of $\varepsilon=k$ requires $n \geq 2 k$.

Adding up results in Eq.(A.5), (A.6), (A.7), we obtain that

$$
\begin{aligned}
\pi\left(r_{u}, \mathbf{r}_{-u}\right) & \leq \frac{\left(\xi_{k}^{+}\right)^{\alpha_{u}} 2^{(k+1)\left(\alpha_{u}+1\right)} k^{(k+2) \alpha_{u}+1}}{\left(\xi_{k}^{-}\right)^{\alpha_{u}+1}} n^{\alpha_{u}-\gamma}+\frac{\left(\xi_{k}^{+}\right)^{\alpha_{u}+1} 2^{(k+1)\left(\alpha_{u}+1\right)+1} k^{(k+2) \alpha_{u}+2}}{\left(\xi_{k}^{-}\right)^{\alpha_{u}+2} \gamma} n^{\alpha_{u}-\gamma}+ \\
& \frac{\left(\xi_{k}^{+}\right)^{\alpha_{u}+1} 2^{\alpha_{u}(k+1)+k+4} k^{\alpha_{u}(k+2)+1}}{\left(\xi_{k}^{-}\right)^{\alpha_{u}+2}} n^{\alpha_{u}-\gamma} \\
& \leq \frac{3\left(\xi_{k}^{+}\right)^{\alpha_{u}+1} \cdot 2^{\alpha_{u}(k+1)+k+4} k^{\alpha_{u}(k+2)+2}}{\gamma\left(\xi_{k}^{-}\right)^{\alpha_{u}+2}} n^{\alpha_{u}-\gamma} \\
& \leq \frac{\left(\xi_{k}^{+}\right)^{\alpha_{u}+1} \cdot 2^{\alpha_{u}(k+1)+k+6} k^{\alpha_{u}(k+2)+2}}{\gamma\left(\xi_{k}^{-}\right)^{\alpha_{u}+2}} n^{\alpha_{u}-\gamma},
\end{aligned}
$$

when $n \geq \max \left\{e^{4}, 2 k\right\}$.

Payoff of $r_{u}>k$. Let $\varepsilon=r_{u}-k(\varepsilon \geq \gamma)$. For this case, we can relax the reciprocity $P_{u}\left(r_{u}, \mathbf{r}_{-u}\right)$ to one and only consider the upper bound on link distance $D\left(r_{u}\right)$. Applying bounds given by inequalities (A.2c), (A.2d) and (A.1a), we obtain:

$$
\begin{aligned}
\pi\left(r_{u}=k+\varepsilon, \mathbf{r}_{-u}\right) \leq D\left(r_{u}\right)^{\alpha_{u}} & \leq \begin{cases}\left(\frac{\xi_{k}^{+} k}{2 \gamma \xi_{k}^{-}} n^{1-\gamma}\right)^{\alpha_{u}} & \text { if } \varepsilon<1, \\
\left(\frac{\xi_{k}^{+}}{\xi_{k}^{-}} \ln (2 k n)\right)^{\alpha_{u}} & \text { if } \varepsilon \geq 1 .\end{cases} \\
& \leq \begin{cases}\left(\frac{\xi_{k}^{+} k}{\xi_{k}^{-} \gamma}\right)^{\alpha_{u}} n^{\alpha_{u}(1-\gamma)} & \text { if } \varepsilon<1, \\
\left(\frac{\xi_{k}^{+}}{\xi_{k}^{-}}\right)^{\alpha_{u}} \ln (2 k n)^{\alpha_{u}} \leq\left(\frac{\xi_{k}^{+}}{\xi_{k}^{-}}\right)^{\alpha_{u}} 2 n^{\alpha_{u}(1-\gamma)} & \text { if } \varepsilon \geq 1 .\end{cases}
\end{aligned}
$$

The last inequality in the above case of $\varepsilon \geq 1$ holds when $n \geq 2 k$ and $\gamma \leq 1 / 2$.

Finally, the lemma holds when we combine Eq.(A.8) and (A.9) 
LEMMA A.4. In the $k$-dimensional DRB game, for sufficiently large n, the payoff of any node $u \in V$ with $\alpha_{u}>0$ in the navigable small world $\mathbf{r} \equiv k$ has the following bounds:

$$
\frac{\left(\xi_{k}^{-}\right)^{\alpha_{u}+1}}{2^{\alpha_{u}}\left(\xi_{k}^{+}\right)^{\alpha_{u}+2}} \frac{n^{\alpha_{u}}}{\ln ^{\alpha_{u}+2}(2 k n)} \leq \pi\left(r_{u}=k, \mathbf{r}_{-u} \equiv k\right) \leq \frac{2^{3+\alpha_{u}}\left(\xi_{k}^{+}\right)^{\alpha_{u}+1}}{\left(\xi_{k}^{-}\right)^{2+\alpha_{u}}} \cdot \frac{n^{\alpha_{u}}}{\ln ^{2+\alpha_{u}} n}
$$

Proof. We have the lower bound for the reciprocity:

$$
P\left(r_{u}, k\right) \geq \frac{\sum_{j=1}^{n / 2} b_{u}(j) \cdot j^{-2 k}}{c^{2}(k)} \geq \frac{\xi_{k}^{-}}{c^{2}(k)} .
$$

Combining the above inequality with bounds (A.2b) and (A.1b), we get.

$$
\pi\left(r_{u}=k, k\right)=D\left(r_{u}\right)^{\alpha_{u}} P\left(r_{u}, k\right) \geq \frac{\left(\xi_{k}^{-}\right)^{\alpha_{u}+1} n^{\alpha_{u}}}{2 c^{\alpha_{u}+2}(k)}=\frac{\left(\xi_{k}^{-}\right)^{\alpha_{u}+1}}{2^{\alpha_{u}}\left(\xi_{k}^{+}\right)^{\alpha_{u}+2}} \frac{n^{\alpha_{u}}}{\ln ^{\alpha_{u}+2}(2 k n)} .
$$

The upper bound on the reciprocity is:

$$
P\left(r_{u}, k\right)=\frac{\sum_{j=1}^{n_{D}} b_{u}(j) \cdot j^{-2 k}}{c^{2}(k)} \leq \frac{\xi_{k}^{+}}{c^{2}(k)}\left(1+\int_{j=1}^{n_{D}} j^{-k-1}\right) \leq \frac{2 \xi_{k}^{+}}{c^{2}(k)}
$$

Combining the above inequality with bounds (A.2b) and (A.1b), we get the upper bound on the payoff:

$$
\pi\left(r_{u}=k, \mathbf{r}_{-u} \equiv k\right)=D\left(r_{u}\right)^{\alpha_{u}} P\left(r_{u}, k\right) \leq \frac{2\left(\xi_{k}^{+}\right)^{\alpha_{u}+1} n^{\alpha_{u}}}{c^{\alpha_{u}+2}(k)} \leq \frac{2^{\alpha_{u}+3}\left(\xi_{k}^{+}\right)^{\alpha_{u}+1}}{\left(\xi_{k}^{-}\right)^{2+\alpha_{u}}} \cdot \frac{n^{\alpha_{u}}}{\ln ^{2+\alpha_{u}} n} .
$$

\section{B. PROOF OF LEMMA 3.1}

LEMMA 3.1. In the $k$-dimensional DRB game, for any constant $\delta$, there exists $n_{0} \in \mathbb{N}$ (may depend on $\delta$ ), for any $n \geq n_{0}$, for any non-zero strategy profile $\mathbf{r} \not \equiv 0$, if a node $w$ satisfies $r_{w} \geq k$ or $r_{w}=\max _{v \in V} r_{v}$, then for any node $u$ within $\delta$ grid distance of $w$ (i.e. $\left.d_{M}(u, w) \leq \bar{\delta}\right), u$ has the unique best response of $r_{u}=k$.

Proof. For a given node $w$, define the set of nodes with distance of $\delta$ to $w$ as: $N_{w, \delta}=$ $\left\{u \mid u \in V \wedge d_{M}(u, w) \leq \delta\right\}$.

In the case of $r_{w} \geq k$, for any $u \in N_{w, \delta}$, if $u$ chooses the strategy $r_{u}=k$, we have:

$$
P\left(r_{u}, \mathbf{r}_{-\mathbf{u}}\right)>p_{u}\left(w, r_{u}\right) p_{w}\left(u, r_{w}\right) \geq \frac{d_{M}(u, w)^{-2 k}}{c(k)^{2}} \geq \frac{\delta^{-2 k}}{c(k)^{2}} .
$$

Combining the above inequality with the bounds in (A.2b) and (A.1b), we get:

$$
\pi\left(r_{u}=k, \mathbf{r}_{-u}\right) \geq D\left(r_{u}\right)^{\alpha_{u}} P\left(r_{u}, \mathbf{r}_{-\mathbf{u}}\right) \geq\left(\frac{\xi_{k}^{-} n}{2 c(k)}\right)^{\alpha_{u}} \cdot \frac{\delta^{-2 k}}{c(k)^{2}} \geq \frac{\left(\xi_{k}^{-}\right)^{\alpha_{u}} \delta^{-2 k}}{2^{\alpha_{u}}\left(\xi_{k}^{+}\right)^{\alpha_{u}+2}} \frac{n^{\alpha_{u}}}{\ln ^{\alpha_{u}+2}(2 k n)} .
$$

However, if node $u$ chooses $r_{u} \neq k$, by Lemma A.3 we know that there is a constant $\kappa$ such that for all sufficiently large $n, \pi\left(r_{u}, \mathbf{r}_{-u}\right) \leq \kappa n^{\alpha_{u}-\min \left\{1, \alpha_{u}\right\} \gamma}$. We see that the lower bound (B.1) for $r_{u}=k$ is in strictly higher order in $n$ than the upper bound of $r_{u} \neq k$, thus there exists $n_{0} \in \mathbb{N}\left(n_{0}\right.$ may depend on $\left.\delta\right)$, such that for all $n \geq n_{0}, r_{u}=k$ is the unique best response to $\mathbf{r}_{-\mathbf{u}}$ for any $u \in N_{w, \delta}$.

In the case of $r_{w}=\max _{v \in V} r_{v}$, if $r_{w} \geq k$, from the above analysis we know that $r_{u}=k$ is the unique best response to $\mathbf{r}_{-\mathbf{u}}$ for any $u \in N_{w, \delta}$.

Otherwise, given $r_{w}<k$, we know $V=V_{<k}$. In this case, we further partition the nodes $V_{<k}$ into two sets: $V_{>0}=\left\{v \in V \mid k>r_{v}>0\right\}$ and $V_{=0}=\left\{v \in V \mid r_{v}=0\right\}$. So we know that:

$$
\pi_{u}(\mathbf{r})=D\left(r_{u}\right)^{\alpha_{u}}\left(P_{u, V_{>0}}(\mathbf{r})+P_{u, V_{=0}}(\mathbf{r})\right) .
$$


Let $r_{\min }$ be the minimum value among the strategies of users in the set of $V=V_{>0}$. Clearly, $\gamma \leq r_{\min } \leq r_{w}<k$.

Payoff of $r_{u}<k$. For any node $u \in N_{w, \beta}$, if it chooses $r_{u}<k$, let $\varepsilon=k-r_{u}(\gamma \leq \varepsilon \leq k)$. Notice that $c(v) \geq c\left(r_{w}\right)$ for any node $v \in V \backslash\{u\}$, so we have:

$$
P_{u, V>0}(\mathbf{r})=\sum_{v \in V>0} \frac{d_{M}(u, v)^{-r_{u}-r_{v}}}{c\left(r_{u}\right) c\left(r_{v}\right)} \leq \sum_{v \in V \backslash\{u\}} \frac{d_{M}(u, v)^{-r_{u}-r_{\min }}}{c\left(r_{u}\right) c\left(r_{w}\right)} \leq \frac{\sum_{j=1}^{n_{D}} \xi_{k}^{+} j^{\varepsilon-1-r_{\text {min }}}}{c\left(r_{u}\right) c\left(r_{w}\right)} .
$$

If $r_{\text {min }}+1 \leq \varepsilon$, we have:

$$
P_{u, V_{>0}}(\mathbf{r}) \leq \xi_{k}^{+} \int_{j=1}^{n_{D}+1} \frac{j^{\varepsilon-r_{\min }-1}}{c\left(r_{u}\right) c\left(r_{w}\right)} \leq \frac{\xi_{k}^{+}\left(n_{D}+1\right)^{\varepsilon-r_{\min }}}{\left(\varepsilon-r_{\min }\right) c\left(r_{u}\right) c\left(r_{w}\right)} \leq \frac{\xi_{k}^{+}(k n)^{\varepsilon-r_{\min }}}{c\left(r_{u}\right) c\left(r_{w}\right)} .
$$

If $r_{\text {min }}+1>\varepsilon$, we have:

$$
P_{u, V_{>0}}(\mathbf{r}) \leq \frac{\xi_{k}^{+}}{c\left(r_{u}\right) c\left(r_{w}\right)}\left(1+\int_{j=1}^{n_{D}} j^{\varepsilon-1-r_{m i n}}\right) \leq \begin{cases}\frac{\xi_{k}^{+}\left((k n)^{\varepsilon-r_{m i n}}+\varepsilon-r_{\min }-1\right)}{\left(\varepsilon-r_{\min }\right) c\left(r_{u}\right) c\left(r_{w}\right)} & \text { if } r_{\text {min }} \neq \varepsilon, \\ \frac{\xi_{k}^{+} \ln (k n)}{c\left(r_{u}\right) c\left(r_{w}\right)} & \text { if } r_{\text {min }}=\varepsilon .\end{cases}
$$

Combining the above bounds on reciprocity with bounds in inequalities (A.4) and (A.1a), we have the payoff of node $u$ gets from $V_{>0}$ :

$$
\begin{aligned}
& D\left(r_{u}\right)^{\alpha_{u}} P_{u, V_{>0}}(\mathbf{r}) \leq\left\{\begin{array}{l}
D\left(r_{u}\right)^{\alpha_{u}} \cdot \frac{\xi_{k}^{+}(k n)^{\varepsilon-r_{m i n}}}{\gamma c\left(r_{u}\right)\left(r_{w}\right)}, \text { if } \varepsilon>r_{m i n}, \\
D\left(r_{u}\right)^{\alpha_{u}} \cdot \frac{\xi_{k}^{+}(n)(k n)}{c\left(r_{u}\right) c\left(r_{w}\right)}, \text { if } \varepsilon=r_{\text {min }}, \\
D\left(r_{u}\right)^{\alpha_{u}} \cdot \frac{\xi_{k}^{+}}{\gamma c\left(r_{u}\right) c\left(r_{w}\right)}, \text { if } \varepsilon<r_{\text {min }} .
\end{array}\right.
\end{aligned}
$$

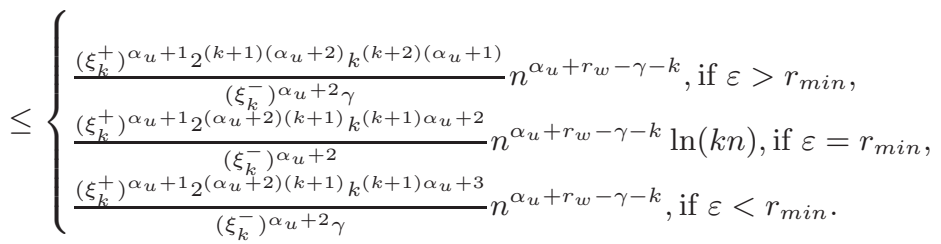

We now consider the payoff of a node $u$ from the set $V_{=0}$. We have:

$$
P_{u, V=0}(\mathbf{r})=\sum_{v \in V_{=0}} \frac{d_{M}(u, v)^{-r_{u}}}{c\left(r_{u}\right) c(0)} \leq \sum_{v \in V \backslash\{u\}} \frac{d_{M}(u, v)^{-r_{u}}}{c\left(r_{u}\right) c(0)}=\frac{1}{c(0)} .
$$

It is easy to get:

$$
c(0)=n^{k}-1 \geq n^{k} / 2 .
$$

Thus, combining with bound (A.4), we have the payoff of node $u$ gets from $V_{=0}$ :

$$
D\left(r_{u}\right)^{\alpha_{u}} P_{u, V=0}(\mathbf{r}) \leq\left(\frac{2^{k+1} \xi_{k}^{+} k^{2+k}}{\xi_{k}^{-}} n\right)^{\alpha_{u}} \cdot \frac{n^{k}}{2} \leq \frac{2^{(k+1) \alpha_{u}+1} k^{(2+k) \alpha_{u}}\left(\xi_{k}^{+}\right)^{\alpha_{u}}}{\left(\xi_{k}^{-}\right)^{\alpha_{u}}} n^{\alpha_{u}-k} .
$$

Combining the above bounds in Eq.(B.3) and Eq.(B.6) with Eq.(B.2), we see the payoff of $r_{u}<k$ in $\mathbf{r}$ is at most $O\left(n^{\alpha_{u}+r_{w}-\gamma-k} \ln (k n)\right)$.

Payoff of $r_{u}>k$. If node $u$ chooses $r_{u}>k$, let $\varepsilon=r_{u}-k \geq \gamma$. We have:

$$
P_{u, V_{>0}}=\sum_{v \in V_{>0}} \frac{d_{M}(u, v)^{-r_{u}-r_{v}}}{c\left(r_{u}\right) c\left(r_{v}\right)} \leq \frac{\sum_{v \in V_{>0}} d_{M}(u, v)^{-r_{u}-r_{v}}}{c\left(r_{u}\right) c\left(r_{w}\right)}<\frac{\sum_{v \in V \backslash\{u\}} d_{M}(u, v)^{-r_{u}}}{c\left(r_{u}\right) c\left(r_{w}\right)}=\frac{1}{c\left(r_{w}\right)} .
$$


Combining the above inequality with bounds (A.2c), (A.2d), (A.1c) and (A.1a) on $D\left(r_{u}\right)$, $c\left(r_{u}\right)$ and $c\left(r_{w}\right)$, respectively, we have the payoff of node $u$ gets from $V_{>0}$ :

$$
D\left(r_{u}\right)^{\alpha_{u}} P_{u, V_{>0}} \leq \begin{cases}\left(\frac{\xi_{k}^{+} k}{2 \gamma \xi_{k}^{-}} n^{1-\gamma}\right)^{\alpha_{u}} \cdot \frac{2^{k+1} k}{\xi_{k}^{-} n^{k-r_{w}}}=\frac{2^{k+1-\alpha_{u}} k^{\alpha_{u}+1}\left(\xi_{k}^{+}\right)^{\alpha_{u}}}{\left(\xi_{k}^{-}\right)^{1+\alpha_{u} \gamma^{\alpha_{u}}} n^{\alpha_{u}+r_{w}-k-\alpha_{u} \gamma}} & \text { if } \varepsilon<1, \\ \left(\frac{\xi_{k}^{+}}{\xi_{k}^{-}} \ln (2 k n)\right)^{\alpha_{u}} \cdot \frac{2^{k+1} k}{\xi_{k}^{-} n^{k-r_{w}}}=\frac{2^{k+1} k\left(\xi_{k}^{+}\right)^{\alpha_{u}}}{\left(\xi_{k}^{-}\right)^{1+\alpha_{u}}} \frac{\ln ^{\alpha_{u}}(2 k n)}{n^{k-r_{w}}} & \text { if } \varepsilon \geq 1 .\end{cases}
$$

Combining the bounds (A.2c), (A.2d), (A.1c) and with bounds Eq.(B.4) and Eq.(B.5), we have the payoff of node $u$ gets from $V_{=0}$ :

$$
D\left(r_{u}\right)^{\alpha_{u}} P_{u, V_{=0}} \leq \begin{cases}\left(\frac{\xi_{k}^{+} k}{2 \gamma \xi_{k}^{-}} n^{1-\gamma}\right)^{\alpha_{u}} \cdot \frac{2}{n^{k}}=2^{1-\alpha_{u}}\left(\frac{\xi_{k}^{+} k}{\xi_{k}^{-} \gamma}\right)^{\alpha_{u}} n^{\alpha_{u}-k-\alpha_{u} \gamma} & \text { if } \varepsilon<1, \\ \left(\frac{\xi_{k}^{+}}{\xi_{k}^{-}} \ln (2 k n)\right)^{\alpha_{u}} \cdot \frac{2}{n^{k}}=2\left(\frac{\xi_{k}^{+}}{\xi_{k}^{-}}\right)^{\alpha_{u}} \frac{\ln ^{\alpha_{u}}(2 k n)}{n^{k}} & \text { if } \varepsilon \geq 1 .\end{cases}
$$

Combining the above bounds in Eq.(B.7) and Eq.(B.8) with Eq.(B.2), we see the payoff of $r_{u}>k$ is at most $O\left(n^{\alpha_{u}+r_{w}-k-\alpha_{u} \gamma}\right)$.

Payoff of $r_{u}=k$. However, if the node $u$ chooses the strategy $r_{u}=k$, we have:

$$
P\left(r_{u}\right)>p_{u}\left(v, r_{u}\right) p_{w}\left(u, r_{w}\right)>\frac{d(u, w)^{-k-r_{w}}}{c(k) c\left(r_{w}\right)}>\frac{\delta^{-2 k}}{c(k) c\left(r_{w}\right)} .
$$

Combining the above inequality with the bounds (A.2b), (A.1b) and (A.1a) on $D\left(r_{u}\right), c(k)$ and $c\left(r_{w}\right)$, we get:

$$
\begin{aligned}
\pi\left(r_{u}=k, \mathbf{r}_{-u}\right) & =D\left(r_{u}\right)^{\alpha_{u}} P\left(r_{u}\right)>\left(\frac{\xi_{k}^{-} n}{2 c(k)}\right)^{\alpha_{u}} \cdot \frac{\delta^{-2 k}}{c(k) c\left(r_{w}\right)}=\frac{\left(\xi_{k}^{-}\right)^{\alpha_{u}} \delta^{-2 k} n^{\alpha_{u}}}{2^{\alpha_{u}} c^{1+\alpha_{u}}(k) c\left(r_{w}\right)} \\
& \geq \frac{\left(\xi_{k}^{-}\right)^{\alpha_{u}-1} k 2^{1+k-\alpha_{u}} \delta^{-2 k}}{\left(\xi_{k}^{+}\right)^{1+\alpha_{u}}} \frac{n^{\alpha_{u}+r_{w}-k}}{\ln ^{1+\alpha_{u}}(2 k n)} .
\end{aligned}
$$

We see that the payoff of $r_{u}=k$ is in strictly higher order in $n$ than the payoff of $r_{u}<k$ or $r_{u}>k$, thus there exists $n_{0} \in \mathbb{N}$ (which may depend on $\delta$ but do not depend on $r_{w}$ since $n^{\alpha_{u}+r_{w}-k}$ is a common term), for all $n \geq n_{0}, r_{u}=k$ is the best response to $\mathbf{r}_{-\mathbf{u}}$ for any $u \in N_{w, \delta}$.

\section{PROOF OF RANDOM SMALL WORLD EQUILIBRIUM}

THEOREM 3.1. For the DRB game in a $k$-dimensional grid, the following is true for sufficiently large $n:{ }^{4}$ For every node $u \in V$, every strategy profile $\mathbf{r}$, and every $s \in \Sigma$, if $\mathbf{r}_{-u} \equiv s$, then $u$ has a unique best response to $\mathbf{r}_{-u} \equiv s$ :

$$
B_{u}\left(\mathbf{r}_{-u} \equiv s\right)= \begin{cases}k & \text { if } s>0 \\ 0 & \text { if } s=0 .\end{cases}
$$

Proof FOR $B_{u}\left(\mathbf{r}_{-u} \equiv s\right)=0$ IF $s=0$. When other players choose strategy $\mathbf{r}_{-u} \equiv 0$, the reciprocity of the player $u$ is constant:

$$
P_{u}\left(r_{u}, \mathbf{r}_{-u} \equiv 0\right)=\sum_{\forall v \neq u} p_{u}\left(v, r_{u}\right) p_{v}\left(u, r_{v}=0\right)=\sum_{\forall v \neq u} \frac{p_{u}\left(v, r_{u}\right)}{|V|-1}=\frac{1}{|V|-1}
$$

\footnotetext{
${ }^{4}$ Technically, a statement being true for sufficiently large $n$ means that there exists a constant $n_{0} \in \mathbb{N}$ that may only depend on model constants such as $k, \gamma$ and $\alpha_{u}$, such that for all $n \geq n_{0}$ the statement is true in the grid with parameter $n$.
} 
Thus, the payoff of the player $u$ is only determined by the link distance $D\left(r_{u}\right)$. Let $X\left(r_{u}\right)$ be the random variable denoting the grid distance from $u$ 's long-range contact to $u$. Then we have $D\left(r_{u}\right)=E\left[X\left(r_{u}\right)\right]$. We want to show the following intuitive claim:

Claim 1. For any $r_{u}<r_{u}^{\prime}, X\left(r_{u}\right)$ strictly stochastically dominates $X\left(r_{u}^{\prime}\right)$, i.e., for all $1 \leq \ell<n_{D}, \operatorname{Pr}\left(X\left(r_{u}\right) \leq \ell\right)<\operatorname{Pr}\left(X\left(r_{u}^{\prime}\right) \leq \ell\right)$.

Proof of the claim. Let $q\left(r_{u}, j\right)$ be the probability that $u$ 's long-range contact is a particular node $v$ at grid distance $j$ from $u$. By definition, $q\left(r_{u}, j\right)=j^{-r_{u}} / c\left(r_{u}\right)$. Then we have $\frac{q\left(r_{u}, j+1\right)}{q\left(r_{u}, j\right)}=\left(\frac{j+1}{j}\right)^{-r_{u}}$. Thus $q\left(r_{u}, j\right)$ is non-increasing in $j$, and the decreasing ratio is faster when $r_{u}$ is larger. Since we know that $\sum_{j=1}^{n} q\left(r_{u}, j\right) b_{u}(j)=1$, it must be that $q\left(r_{u}, 1\right)<q\left(r_{u}^{\prime}, 1\right), q\left(r_{u}, n_{D}\right)>q\left(r_{u}^{\prime}, n_{D}\right)$, and there exists a $\bar{j}$ such that for all $j \leq \bar{j}$, $q\left(r_{u}, j\right) \leq q\left(r_{u}^{\prime}, j\right)$, and for all $j>\bar{j}, q\left(r_{u}, j\right)>q\left(r_{u}^{\prime}, j\right)$.

By the definition of $X\left(r_{u}\right)$, we have $\operatorname{Pr}\left(X\left(r_{u}\right) \leq \ell\right)=\sum_{j=1}^{\ell} q\left(r_{u}, j\right) b_{u}(j)$. Thus, for any $1 \leq \ell \leq \bar{j}, \operatorname{Pr}\left(X\left(r_{u}\right) \leq \ell\right)=\sum_{j=1}^{\ell} q\left(r_{u}, j\right) b_{u}(j)<\sum_{j=1}^{\ell} q\left(r_{u}^{\prime}, j\right) b_{u}(j)=\operatorname{Pr}\left(X\left(r_{u}^{\prime}\right) \leq \ell\right)$. For any $\bar{j}<\ell<n_{D}, \operatorname{Pr}\left(X\left(r_{u}\right) \leq \ell\right)=\sum_{j=1}^{\ell} q\left(r_{u}, j\right) b_{u}(j)=1-\sum_{j=\ell+1}^{n_{D}} q\left(r_{u}, j\right) b_{u}(j)<$ $1-\sum_{j=\ell+1}^{n_{D}} q\left(r_{u}^{\prime}, j\right) b_{u}(j)=\operatorname{Pr}\left(X\left(r_{u}^{\prime}\right) \leq \ell\right)$. Therefore, we have the claim that $X\left(r_{u}\right)$ strictly stochastically dominates $X\left(r_{u}^{\prime}\right)$.

With this claim, we immediately have $E\left[X\left(r_{u}\right)\right]>E\left[X\left(r_{u}^{\prime}\right)\right]$. As a consequence, $D(0)=$ $E[X(0)]>E\left[X\left(r_{u}^{\prime}\right)\right]=D\left(r_{u}^{\prime}\right)$ for any $r_{u}^{\prime}>0$. Therefore, $r_{u}=0$ is the player $u$ 's unique best response to $\mathbf{r}_{-u} \equiv 0$.

\section{PROOF OF THEOREM 3.3}

THEOREM 3.3. For the DRB game in the $k$-dimensional grid, the navigable small-world network $(\mathbf{r} \equiv k)$ is a strong Nash equilibrium for sufficiently large $n$.

PROOF. We actually prove a slightly stronger result: any node $u$ in any strategy profile $\mathbf{r}$ with $r_{u} \neq k$ is strictly worse off than its payoff in the navigable equilibrium, when $n$ is large enough. With the Lemma A.4, we see that a player $u$ has the payoff at least $\Omega\left(\frac{n^{\alpha u}}{\ln ^{2+\alpha u}(2 k n)}\right)$ before derivation. Suppose that a coalition $C$ deviates, and the new strategy profile is $\mathbf{r}$. Then some node $u \in C$ must select a new $r_{u} \neq k$. By Lemma A.3, there is a constant $\kappa$ such that for all sufficiently large $n, \pi\left(r_{u}, \mathbf{r}_{-u}\right) \leq \kappa n^{\alpha_{u}-\min \left\{1, \alpha_{u}\right\} \gamma}$. Thus we see that the payoff of $u$ before the deviation is in strictly higher order in $n$ than its payoff after the deviation. Therefore, for all sufficiently large $n, u$ is strictly worse off, which means no coalition could make some member strictly better off while others not worse off. Hence, navigable small-world network $(\mathbf{r} \equiv k)$ is a strong Nash equilibrium.

\section{E. PROOF OF THEOREM 3.4}

THEOREM 3.4. For the DRB game in a $k$-dimensional grid, the random small-world $N E \mathbf{r} \equiv 0$ is not a 2-strong Nash equilibrium for sufficiently large $n$. have:

Proof. Given a pair of grid neighbors $(u, v)$, if they both choose the strategy $k$, we

$$
P\left(r_{u}, \mathbf{r}_{-\mathbf{u}}\right)>p_{u}\left(v, r_{u}\right) p_{v}\left(u, r_{u}\right) \geq \frac{d_{M}(u, w)^{-2 k}}{c(k)^{2}} \geq \frac{1}{c(k)^{2}} .
$$

Combining the above inequality with the bounds (A.2b) and (A.1b), we get:

$$
\pi\left(r_{u}=k, \mathbf{r}_{-u}\right)=D\left(r_{u}\right)^{\alpha_{u}} P\left(r_{u}, \mathbf{r}_{-\mathbf{u}}\right)>\left(\frac{\xi_{k}^{-} n}{2}\right)^{\alpha_{u}} \frac{1}{c^{2+\alpha_{u}}(k)} \geq \frac{\left(\xi_{k}^{-}\right)^{\alpha_{u}}}{2^{\alpha_{u}}\left(\xi_{k}^{+}\right)^{\alpha_{u}+2}} \frac{n^{\alpha_{u}}}{\ln ^{\alpha_{u}+2}(2 k n)} .
$$

However, if node $u$ chooses $r_{u} \neq k$, by Lemma A.3 we know that there is a constant $\kappa$ such that for all sufficiently large $n, \pi\left(r_{u}, \mathbf{r}_{-u}\right) \leq \kappa n^{\alpha_{u}-\min \left\{1, \alpha_{u}\right\} \gamma}$. We see that the payoff of 
$r_{u}=k$ is in strictly higher order in $n$ than its original payoff. Notice that the proof for the increase of node $v$ 's payoff is similar to that of node $u$, so both colluding nodes get strictly higher payoff. The theorem is proved.

\section{F. PROOF OF THEOREM 3.6}

FACT 1. (Chernoff bound). Let $X$ be a sum of $n$ independent random variables $\left\{X_{i}\right\}$, with $E\left[X_{i}\right]=\mu ; X_{i} \in\{0,1\}$ for all $i \leq n$. For any $0<\epsilon<1$,

$$
\operatorname{Pr}[X \leq(1-\epsilon) \mu] \leq e^{-\frac{\mu \epsilon^{2}}{2}}, \operatorname{Pr}[X \geq(1+\epsilon) \mu] \leq e^{-\frac{\epsilon^{2}}{2+\epsilon} \mu} .
$$

Based on the Chernoff bound, we have the following lemma. Let $Y_{u}(j, s)$ be the number of players with grid distance $j$ to $u$ and a strategy of $s$.

Lemma F.1. In the $k$-dimensional DRB game $(k>1)$, for any $\eta>0$, if each player chooses a strategy $s$ independently with probability $p_{s} \geq \eta$ from a finite strategy set $S \subseteq \Sigma$, then for all $n \geq|S|$, with probability $1-1 / n$, the following property holds:

$$
Y_{u}(j, s)>\frac{\eta b_{u}(j)}{2}, \forall u \in V, \forall s \in S, \forall j \in \mathbb{N} \cap\left[\rho\left(\frac{\ln n}{\eta}\right)^{\frac{1}{k-1}}, \frac{n}{2}\right],
$$

where $\rho=\left(\frac{24+8 k}{\xi_{k}^{-}}\right)^{\frac{1}{k-1}}$ is a constant.

Proof. Since individual players choose strategy of $s$ independently with probability $p_{s}$, $E\left[Y_{u}(j, s)\right]=p_{s} b_{u}(j) \geq \eta b_{u}(j)$. Based on the Chernoff bound, we have:

$$
P\left(Y_{u}(j) \leq(1-\epsilon) E\left[Y_{u}(j, s)\right]\right) \leq \exp \left(-\frac{\epsilon^{2} E\left[Y_{u}(j, s)\right]}{2}\right) \leq \exp \left(-\frac{\epsilon^{2} \eta b_{u}(j)}{2}\right) .
$$

Note that $b_{u}(j) \geq \xi_{k}^{-} j^{k-1}$ for $0<j \leq\lfloor n\rfloor / 2$. Let $m=|S|$. Let $\varrho=\left(\frac{(16+8 k) \ln n+8 \ln m}{\eta \xi_{k}^{-}}\right)^{\frac{1}{k-1}}$ For $\varrho \leq j \leq\lfloor n\rfloor / 2$, we have:

$$
P\left(Y_{u}(j) \leq \frac{\eta b_{j}(u)}{2}\right) \leq \frac{1}{m n^{k+2}} .
$$

Since there are $n^{k}$ players in the $k$ dimensional grid, by union bound, we have $\forall u, \forall s$, for any $\varrho \leq j \leq\lfloor n\rfloor / 2$,

$$
P\left(Y_{u}(j)>\frac{\eta b_{j}(u)}{2}\right) \geq 1-\frac{1}{n},
$$

As $m$ is a constant, we can rewrite $\varrho$ as:

$$
\varrho=\left(\frac{(16+8 k) \ln n+8 \ln m}{\eta \xi_{k}^{-}}\right)^{\frac{1}{k-1}} \leq\left(\frac{24+8 k}{\xi_{k}^{-}}\right)^{\frac{1}{k-1}}\left(\frac{\ln n}{\eta}\right)^{\frac{1}{k-1}},
$$

holds for all $n \geq m$.

THEOREM 3.6. Consider the navigable small-world equilibrium $\mathbf{r} \equiv k$ for the DRB game in a $k$-dimensional grid $(k>1)$. Suppose that with probability $p_{u}$ each node $u \in V$ independently perturbs $r_{u}$ to an arbitrary strategy $r_{u}^{\prime} \in \Sigma$, and with probability $1-p_{u} r_{u}^{\prime}=r_{u}$. Let $\alpha_{\min }=\min _{u \in V} \alpha_{u}$, then for any constant $\varepsilon$ with $0<\varepsilon<\min \left\{1, \alpha_{\min }\right\} \gamma / 4$, there exists $n_{0} \in \mathbb{N}$ (depending only on $k, \gamma$, and $\varepsilon$ ), for all $n \geq n_{0}$, if $p_{u} \leq 1-n^{-\varepsilon}$, with probability at least $1-1 / n$, the perturbed strategy profile $\mathbf{r}^{\prime}$ moves back to the navigable small world $(\mathbf{r} \equiv k)$ in one synchronous step, or as soon as every node takes at least one asynchronous step in the best response dynamics. 
Proof. Given a deviation probability $p_{u} \leq 1-n^{-\varepsilon}$ for node $u$, we know that node $u$ still uses the original strategy $k$ with a probability of $1-p_{u} \geq n^{-\varepsilon}$. By Lemma F.1 we know that, with probability $1-\frac{1}{n}$, the following property holds:

$$
Y_{u}(j, k)>\frac{n^{-\varepsilon} b_{u}(j)}{2}, \forall u \in V, \forall j \in \mathbb{N} \cap\left[\rho\left(\frac{\ln n}{n^{-\varepsilon}}\right)^{\frac{1}{k-1}}, \frac{n}{2}\right] .
$$

When the above property holds, we fix any node $u$ and examine its payoff. In the case of $r_{u}=k$, the reciprocity that $u$ gets from those still choosing strategy of $k$ is:

$$
\begin{aligned}
P_{u, V_{=k}}(\mathbf{r}) & \geq \frac{n^{-\varepsilon}}{2} \frac{\sum_{j=\rho\left(\frac{\ln n}{n^{-\varepsilon}}\right)^{\frac{1}{k-1}} b_{u}(j) \cdot j^{-2 k}}^{c^{2}(k)} \geq \frac{\xi_{k}^{-} n^{-\varepsilon}}{2} \frac{\sum_{j=\rho\left(\frac{\ln n}{n^{-\varepsilon}}\right)^{\frac{1}{k-1}} j^{-k-1}}^{c^{2}(k)}}{c^{2}(k)} \geq \frac{\xi_{k}^{-} n^{-4 \varepsilon}}{2 c^{2}(k) \rho^{3} \ln ^{3} n} .}{} \\
& \geq \frac{\xi_{k}^{-} n^{-\varepsilon}}{2} \frac{\rho^{\frac{-(k+1)}{k-1}}\left(\frac{\ln n}{n^{-\varepsilon}}\right)^{\frac{-(k+1)}{k-1}}}{c^{2}}
\end{aligned}
$$

The last inequality holds as $k \geq 2$.

Combing with the above bound with bounds (A.2b) and (A.1b), we get:

$$
\begin{aligned}
\pi_{u}\left(r_{u}=k, \mathbf{r}\right) & \geq D\left(r_{u}\right)^{\alpha_{u}} P_{u, V_{=k}}(\mathbf{r}) \geq\left(\frac{\xi_{k}^{-} n}{2 c(k)}\right)^{\alpha_{u}} \cdot \frac{\xi_{k}^{-} n^{-4 \varepsilon}}{2 c^{2}(k) \rho^{3} \ln ^{3} n} \\
& \geq \frac{\left(\xi_{k}^{-}\right)^{\alpha_{u}+1}}{2^{\alpha_{u}+1}\left(\xi_{k}^{+}\right)^{\alpha_{u}+2} \rho^{3}} \frac{n^{\alpha_{u}-4 \varepsilon}}{\ln ^{2+\alpha_{u}}(2 k n) \cdot \ln ^{3}(n)} \geq \frac{\left(\xi_{k}^{-}\right)^{\alpha_{u}+1}}{2^{\alpha_{u}+1}\left(\xi_{k}^{+}\right)^{\alpha_{u}+2} \rho^{3}} \frac{n^{\alpha_{u}-4 \varepsilon}}{\ln ^{5+\alpha_{u}}(2 k n)} .
\end{aligned}
$$

By Lemma A.3, there is a constant $\kappa$ such that for all sufficiently large $n, \pi\left(r_{u} \neq k, \mathbf{r}_{-u}\right) \leq$ $\kappa n^{\alpha_{u}-\min \left\{1, \alpha_{u}\right\} \gamma}$. Comparing with Eq. (F.2), since $\alpha_{\min } \leq \alpha_{u}$ for any node $u$ and $\varepsilon<$ $\min \left\{1, \alpha_{\min }\right\} \gamma / 4$, the payoff of $u$ with strategy $r_{u}=k$ is in strictly higher order in $n$ than its payoff after the deviation. Therefore, when the property Eq. (F.1) holds, for all sufficiently large $n, u$ get strictly better payoff than any other strategy choice by choosing $r_{u}=k$ after the deviation.

Therefore, when the property Eq. (F.1) holds, the perturbed strategy profile $\mathbf{r}^{\prime}$ moves back to the navigable small world $(\mathbf{r} \equiv k)$ in one synchronous step, as every player $u$ moves from its current strategy to its best response $r_{u}=k$. Also, it is clear that the property Eq. (F.1) consistently holds as any player takes one asynchronous step. This is because the asynchronous move only increases the number of nodes choosing the strategy of $k$, so the best response of every player is always $k$ after every asynchronous step. Thus, the perturbed strategy profile moves back to the navigable small world as soon as every node takes at least one asynchronous step. Notice that the property Eq. (F.1) holds with a probability of $1-1 / n$, so the theorem is proved.

\section{G. PROOF OF THEOREM 3.7}

THEOREM 3.7. For the DRB game in a $k$-dimensional grid $(k>1)$ with the initial strategy profile $\mathbf{r} \equiv 0$ and a finite perturbed strategy set $S \subset \Sigma$ with at least one non-zero entry $(0<\max S \leq \beta)$, for any constant $\varepsilon$ with $0<\varepsilon<\gamma / 2$, there exists $n_{0} \in \mathbb{N}$ (depending only on $k, \gamma$, and $\varepsilon$ ), for all $n \geq n_{0}$, if for any $u \in V$, with independent probability of $p \geq n^{-\frac{(k-1) \varepsilon}{k+\beta}}, r_{u} \in S \backslash\{0\}$ after the perturbation, then with probability at least $1-1 / n$, the network converges to the navigable small world in one synchronous step, or as soon as every node takes at least one asynchronous step in the best response dynamics.

Proof. Fix any node $u \in V$. Let $\mathbf{r}$ be the strategy profile after perturbation. We partition nodes in $V \backslash\{u\}$ into sets $V_{s}, s \in S \cup\{0\}$, where $V_{s}=\left\{v \in V \backslash\{u\} \mid r_{v}=s\right\}$. Let 
$P_{u, V_{s}}(\mathbf{r})$ be the reciprocity $u$ obtained from subset $V_{s}$. Then we have

$$
\pi\left(r_{u}, \mathbf{r}_{-u}\right)=D\left(r_{u}\right) \cdot \sum_{s \in S \cup\{0\}} P_{u, V_{s}}(\mathbf{r}) .
$$

For any node $u$ and any given $s \in S \cup\{0\}$, we now compare the payoff it gets from $V_{s}$ when using $r_{u}=k$ and $r_{u}=s^{\prime} \neq k$, respectively.

$$
\left(\frac{D\left(r_{u}=s^{\prime}\right)}{D\left(r_{u}=k\right)}\right)^{\alpha_{u}} \cdot \frac{P_{u, V_{s}}(\mathbf{r})}{P_{u, V_{s}}(\mathbf{r})}=\left(\frac{D\left(r_{u}=s^{\prime}\right)}{D\left(r_{u}=k\right)}\right)^{\alpha_{u}} \cdot \frac{\sum_{v \in V_{s}} \frac{d(u, v)-s^{\prime}-s}{c\left(s^{\prime}\right) c(s)}}{\sum_{v \in V_{s}} \frac{d(u, v)-k-s}{c(k) c(s)}} .
$$

For a given node $u$ and a subset of nodes $\Gamma$, let define $d_{\min , \Gamma}$ and $d_{\max , \Gamma}$ be the minimum and maximum grid distances between node $u$ and any node $v \in \Gamma$, respectively. In other words, $d_{\min , \Gamma} \leq d_{M}(u, v) \leq d_{\max , \Gamma}, \forall v \in \Gamma$. With this definition, for any $v \in V_{s}$, we have:

$$
\frac{\frac{d(u, v)-s^{\prime}-s}{c\left(s^{\prime}\right) c(s)}}{\frac{d(u, v)-k-s}{c(k) c(s)}}=\frac{c(k)}{c\left(s^{\prime}\right)} d(u, v)^{k-s^{\prime}} \leq\left\{\begin{array}{ll}
\frac{c(k)}{c\left(s^{\prime}\right)} d_{\max , V_{s}}^{k-s^{\prime}} & \text { if } s^{\prime}<k, \\
\frac{c(k)}{c\left(s^{\prime}\right)} d_{\min , V_{s}}^{k-s^{\prime}} & \text { if } s^{\prime}>k .
\end{array} .\right.
$$

Combing the above inequality, we have:

$$
\left(\frac{D\left(r_{u}=s^{\prime}\right)}{D\left(r_{u}=k\right)}\right)^{\alpha_{u}} \cdot \frac{P_{u, V_{s}}(\mathbf{r})}{P_{u, V_{s}}(\mathbf{r})} \leq\left\{\begin{array}{ll}
\left(\frac{D\left(r_{u}=s^{\prime}\right)}{D\left(r_{u}=k\right)}\right)^{\alpha_{u}} \cdot \frac{c(k)}{c\left(s^{\prime}\right)} d_{\max , V_{s}}^{k-s^{\prime}} & \text { if } s^{\prime}<k, \\
\left(\frac{D\left(r_{u}=s^{\prime}\right)}{D\left(r_{u}=k\right)}\right)^{\alpha_{u}} \cdot \frac{c(k)}{c\left(s^{\prime}\right)} d_{\min , V_{s}}^{k-s^{\prime}} & \text { if } s^{\prime}>k .
\end{array} .\right.
$$

We first show that $\pi\left(r_{u}=k, \mathbf{r}_{-u}\right)>\pi\left(r_{u}=s^{\prime}, \mathbf{r}_{-u}\right)$ when $s^{\prime}>k$. In the case of $s^{\prime}>k$, as $d_{\min , V_{s}} \geq 1$, combining the above inequality with the bounds (A.2c), (A.2b), (A.1c) and (A.1b) on $D\left(s^{\prime}\right), D(k), c\left(s^{\prime}\right)$ and $c(k)$, we get:

$$
\left(\frac{D\left(r_{u}=s^{\prime}\right)}{D\left(r_{u}=k\right)}\right)^{\alpha_{u}} \cdot \frac{P_{u, V_{s}}(\mathbf{r})}{P_{u, V_{s}}(\mathbf{r})}=O\left(\left(\frac{n^{1-\gamma}}{n}\right)^{\alpha_{u}} \cdot c(k)^{\alpha_{u}+1}\right)=O\left(\frac{\ln ^{\alpha_{u}+1}(2 k n)}{n^{\alpha_{u} \gamma}}\right) .
$$

Therefore, we can find a constant $\sigma$ such that:

$$
\begin{aligned}
& \pi\left(r_{u}=k, \mathbf{r}_{-u}\right)-\pi\left(r_{u}=s^{\prime}, \mathbf{r}_{-u}\right)=\sum_{s \in S \cup\{0\}}\left[D\left(r_{u}=k\right)^{\alpha_{u}} P_{u, V_{s}}(\mathbf{r})-D\left(r_{u}=s^{\prime}\right)^{\alpha_{u}} P_{u, V_{s}}(\mathbf{r})\right] \\
& =\sum_{s \in S \cup\{0\}} D\left(r_{u}=k\right)^{\alpha_{u}} P_{u, V_{s}}(\mathbf{r})\left(1-\frac{D\left(r_{u}=s^{\prime}\right)^{\alpha_{u}} P_{u, V_{s}}(\mathbf{r})}{D\left(r_{u}=k\right)^{\alpha_{u}} P_{u, V_{s}}(\mathbf{r})}\right) \\
& \geq \sum_{s \in S \cup\{0\}} D\left(r_{u}=k\right)^{\alpha_{u}} P_{u, V_{s}}(\mathbf{r})\left(1-\frac{\ln ^{\alpha_{u}+1}(2 k n)}{n^{\alpha_{u} \gamma}}\right)>0,
\end{aligned}
$$

for sufficiently large $n$.

We next show that $\pi\left(r_{u}=k, \mathbf{r}_{-u}\right)>\pi\left(r_{u}=s^{\prime}, \mathbf{r}_{-u}\right)$ when $s^{\prime}<k$. Note here we require the constant $\varepsilon<\gamma / 2$ in the theorem. We first find a distance threshold to partition nodes into nodes nearby to $u$ and nodes far away from $u$. We want to prove that $\pi\left(r_{u}=k, \mathbf{r}_{-u}\right)-\pi\left(r_{u}=\right.$ $\left.s^{\prime}, \mathbf{r}_{-u}\right)$ is dominated by the nearby nodes.

In the case of $s^{\prime}<k$, we can find a constant $\nu=1-\frac{\gamma-2 \varepsilon}{2 k}$ such that, for any $s \in S$, the set $V_{s}$ can be partitioned into two subsets: (i) $V_{s}^{-}=\left\{v \in V \mid r_{v}=s \wedge d_{M}(u, v) \leq n^{\nu}\right\}$, and (ii) $V_{s}^{+}=\left\{v \in V \mid r_{v}=s \wedge d_{M}(u, v)>n^{\nu}\right\}$. Notice that $d_{\max , V_{s}^{-}}$is at most $n^{\nu}$. Combining the above inequality Eq. (G.2) with the bounds (A.2a), (A.2b), (A.1a) and (A.1b) on $D\left(s^{\prime}\right)$, $D(k), c\left(s^{\prime}\right)$ and $c(k)$, we get:

$$
\left(\frac{D\left(r_{u}=s^{\prime}\right)}{D\left(r_{u}=k\right)}\right)^{\alpha_{u}} \cdot \frac{P_{u, V_{s}^{-}}(\mathbf{r})}{P_{u, V_{s}^{-}}(\mathbf{r})}=O\left(\frac{c(k)^{\alpha_{u}+1}}{n^{\left(k-s^{\prime}\right)(1-\nu)}}\right)=O\left(\frac{\ln ^{\alpha_{u}+1}(2 k n)}{n^{(1-\nu) \gamma}}\right),
$$


where $\nu<1$.

Notice that $P_{u, V_{s}}=P_{u, V_{s}^{-}}+P_{u, V_{s}^{+}}$. Based on the bound in Eq. (G.5), we can find a constant $\sigma^{\prime}$ such that:

$$
\begin{aligned}
& \pi\left(r_{u}=k, \mathbf{r}_{-u}\right)-\pi\left(r_{u}=s^{\prime}, \mathbf{r}_{-u}\right) \\
& \geq \sum_{s \in S \cup\{0\}} D\left(r_{u}=k\right)^{\alpha_{u}} P_{u, V_{s}^{-}}(\mathbf{r})-\sum_{s \in S \cup\{0\}} D\left(r_{u}=s^{\prime}\right)^{\alpha_{u}} P_{u, V_{s}}(\mathbf{r}) \\
& \geq \sum_{s \in S \cup\{0\}} D\left(r_{u}=k\right)^{\alpha_{u}} P_{u, V_{s}^{-}}(\mathbf{r})\left(1-\frac{D\left(r_{u}=s^{\prime}\right)^{\alpha_{u}} P_{u, V_{s}^{-}}(\mathbf{r})}{D\left(r_{u}=k\right)^{\alpha_{u}} P_{u, V_{s}^{-}}(\mathbf{r})}\right)-\sum_{s \in S \cup\{0\}} D\left(r_{u}=s^{\prime}\right)^{\alpha_{u}} P_{u, V_{s}^{+}}(\mathbf{r}) \\
& \geq \sum_{s \in S \cup\{0\}} D\left(r_{u}=k\right)^{\alpha_{u}} P_{u, V_{s}^{-}}(\mathbf{r})\left(1-\frac{\sigma^{\prime} \ln ^{\alpha_{u}+1}(2 k n)}{n^{(1-\nu) \gamma}}\right)-\sum_{s \in S \cup\{0\}} D\left(r_{u}=s^{\prime}\right)^{\alpha_{u}} P_{u, V_{s}^{+}}(\mathbf{r}) \\
& \geq \sum_{s \in S \cup\{0\}} \frac{D\left(r_{u}=k\right)^{\alpha_{u}} P_{u, V_{s}^{-}}(\mathbf{r})}{2}-\sum_{s \in S \cup\{0\}} D\left(r_{u}=s^{\prime}\right)^{\alpha_{u}} P_{u, V_{s}^{+}}(\mathbf{r})
\end{aligned}
$$

for sufficiently large $n$.

We now give the lower bound of the first term $D\left(r_{u}=k\right)^{\alpha_{u}} P_{u, V_{s}^{-}}(\mathbf{r})$. Let $U_{j}=\{v \mid$ $\left.d_{M}(u, v)=j \wedge r_{v}>0\right\}$. By Lemma F.1, with probability $1-1 / n$,

$$
\left|U_{j}\right|>\frac{\eta b_{u}(j)}{2}, \forall u \in V, \forall j \in \mathbb{N} \cap\left[\rho\left(\frac{\ln n}{\eta}\right)^{\frac{1}{k-1}}, \frac{n}{2}\right] .
$$

For $j=\left\lceil\rho\left(\frac{\ln n}{\eta}\right)^{\frac{1}{k-1}}\right\rceil$, we have:

$$
\begin{aligned}
P_{u, U_{j}}(\mathbf{r}) & =\sum_{v \in U_{j}} p_{u}\left(v, r_{u}\right) \cdot p_{v}\left(u, r_{v}\right)=\sum_{v \in U_{j}} \frac{j^{-k}}{c(k)} \cdot \frac{j^{-r_{v}}}{c\left(r_{v}\right)} \geq \sum_{v \in U_{j}} \frac{j^{-k}}{c(k)} \cdot \frac{j^{-\beta}}{c(\gamma)} \geq \frac{\eta \cdot b_{u}(j) \cdot j^{-k-\beta}}{2 c(k) c(\gamma)} \\
& \geq \frac{\eta \cdot \xi_{k}^{-} j^{k-1} \cdot j^{-k-\beta}}{2 c(k) c(\gamma)} \geq \frac{\eta \xi_{k}^{-} \rho^{\frac{-(\beta+1)}{k-1}}\left(\frac{\ln n}{\eta}\right)^{\frac{-(\beta+1)}{k-1}}}{2 c(k) c(\gamma)} .
\end{aligned}
$$

We now fix $\eta=1 / n^{\frac{(k-1) \varepsilon}{k+\beta}}(0<\varepsilon<\gamma / 2)$, and have:

$$
P_{u, U_{j}}(\mathbf{r}) \geq \frac{\xi_{k}^{-}}{2 \rho^{\frac{\beta+1}{k-1}} c(k) c(\gamma)} \cdot \frac{1}{(\ln n)^{\frac{(\beta+1)}{k-1}} n^{\varepsilon}} .
$$

Note that $U_{j} \subseteq \cup_{s \in S \backslash\{0\}} V_{s}^{-}$, since $j=\left\lceil\rho\left(\frac{\ln n}{\eta}\right)^{\frac{1}{k-1}}\right\rceil=\left\lceil\rho \ln \frac{1}{k-1} n \cdot n^{\frac{\varepsilon}{k+\beta}}\right\rceil<n^{\frac{k-\gamma / 2+\varepsilon}{k}}=$ $n^{\nu}$ for sufficiently large $n$. Combining with the bounds (A.2b), (A.1a) and (A.1b) on $D(k)$, $c(\gamma)$ and $c(k)$, we get:

$$
\begin{aligned}
D\left(r_{u}=k\right)^{\alpha_{u}} \sum_{s \in S \backslash\{0\}} P_{u, V_{s}^{-}} & \geq D\left(r_{u}=k\right)^{\alpha_{u}} P_{u, U_{j}}\left(r_{u}=k, \mathbf{r}_{-u}\right) \\
& =\Omega\left(\frac{n^{\alpha_{u}}}{\ln ^{\alpha_{u}}(2 k n)} \cdot \frac{1}{\ln (2 k n) \cdot n^{k-\gamma}} \cdot \frac{1}{(\ln n)^{\frac{(\beta+1)}{k-1}} n^{\varepsilon}}\right)=\Omega\left(\frac{n^{\alpha_{u}-k-\varepsilon+\gamma}}{\ln ^{a}(2 k n)}\right),
\end{aligned}
$$

where $a=\alpha_{u}+1+\frac{\beta+1}{k-1}$ is constant. 
We next give the upper bound of the second term $D\left(r_{u}=s^{\prime}\right)^{\alpha_{u}} P_{u, V_{s}^{+}}$. Notice that $d_{M}(u, v)>n^{\nu}$ for any $v$ in $V_{s}^{+}$, so for any $s$, we have:

$$
P_{u, V_{s}^{+}}(\mathbf{r})=\sum_{v \in V_{s}^{+}} \frac{d_{M}(u, v)^{-s^{\prime}-s}}{c\left(s^{\prime}\right) c(s)} \leq \sum_{v \in V_{s}^{+}} \frac{n^{-\nu\left(s^{\prime}+s\right)}}{c\left(s^{\prime}\right) c(s)} .
$$

In the case of $s<k$, combining the above inequality with the bound (A.1a) on $c\left(s^{\prime}\right)$ and $c(s)$, we get:

$$
P_{u, V_{s}^{+}}(\mathbf{r}) \leq \sum_{v \in V_{s}^{+}} \frac{2^{2 k+2} k^{2}}{\left(\xi_{k}^{-}\right)^{2}} n^{\left(s+s^{\prime}\right)(1-\nu)-2 k} \leq \sum_{v \in V_{s}^{+}} \frac{2^{2 k+2} k^{2}}{\left(\xi_{k}^{-}\right)^{2}} n^{2 k(1-\nu)-2 k}=\left|V_{s}^{+}\right| \frac{2^{2 k+2} k^{2}}{\left(\xi_{k}^{-}\right)^{2}} n^{-2 k \nu} .
$$

In the other case of $s \geq k$, combining the inequality Eq. (G.10) with the bounds (A.1a), (A.1c) on $c\left(s^{\prime}\right)$ and $c(s)$, respectively, we get:

$$
\begin{aligned}
P_{u, V_{s}^{+}}(\mathbf{r}) & \leq \sum_{v \in V_{s}^{+}} \frac{n^{-\nu\left(s^{\prime}+k\right)}}{c\left(s^{\prime}\right) \xi_{k}^{-}} \leq \sum_{v \in V_{s}^{+}} \frac{2^{k+1} k}{\left(\xi_{k}^{-}\right)^{2}} n^{(1-\nu) s^{\prime}-(1+\nu) k} \leq \sum_{v \in V_{s}^{+}} \frac{2^{k+1} k}{\left(\xi_{k}^{-}\right)^{2}} n^{(1-\nu) k-(1+\nu) k} \\
& =\left|V_{s}^{+}\right| \frac{2^{k+1} k}{\left(\xi_{k}^{-}\right)^{2}} n^{-2 k \nu} .
\end{aligned}
$$

Combining the above inequalities Eq. (G.11) and Eq. (G.12) with the bound (A.2b) on distance $D\left(s^{\prime}\right)$, we know that for any $s$ :

$$
D\left(r_{u}=s^{\prime}\right)^{\alpha_{u}} P_{u, V_{s}^{+}}=O\left(\left|V_{s}^{+}\right| n^{\alpha_{u}-2 k \nu}\right) .
$$

We are now ready to combine the above bounds and show that $\pi\left(r_{u}=k, \mathbf{r}_{-u}\right)>\pi\left(r_{u}=\right.$ $\left.s^{\prime}, \mathbf{r}_{-u}\right)$ when $s^{\prime}<k$. More specifically, combining the inequalities in Eq. (G.6), Eq. (G.9) and Eq. (G.13), we get:

$$
\begin{aligned}
& \pi\left(r_{u}=k, \mathbf{r}_{-u}\right)-\pi\left(r_{u}=s^{\prime}, \mathbf{r}_{-u}\right) \\
& \geq \sum_{s \in S \backslash\{0\}} \frac{D\left(r_{u}=k\right)^{\alpha_{u}} P_{u, V_{s}^{-}}^{-}}{2}-\sum_{s \in S \cup\{0\}} D\left(r_{u}=s^{\prime}\right)^{\alpha_{u}} P_{u, V_{s}^{+}}, \\
& \geq \frac{\rho n^{\alpha_{u}-k+\gamma-\varepsilon}}{2 \ln ^{a}(2 k n)}-\rho^{\prime}\left|\cup_{s \in S \cup\{0\}} V_{s}^{+}\right| \cdot n^{\alpha_{u}-2 k \nu} \geq \frac{\rho n^{\alpha_{u}-k+\gamma-\varepsilon}}{2 \ln ^{a}(2 k n)}-\rho^{\prime} n^{k} \cdot n^{\alpha_{u}-2 k+\gamma-2 \varepsilon} \\
& \geq \frac{\rho n^{\alpha_{u}-k+\gamma-\varepsilon}}{2 \ln ^{a}(2 k n)}-\rho^{\prime} n^{\alpha_{u}-k+\gamma-2 \varepsilon}
\end{aligned}
$$

where $\sigma, \rho, \rho^{\prime}, a$ are all constants.

As $0<\varepsilon<\gamma / 2$, the first term in Eq. (G.14) is in strictly higher order in $n$ than the second term in Eq. (G.14), we know that for sufficiently large $n, \pi\left(r_{u}=k, \mathbf{r}_{-u}\right)>\pi\left(r_{u}=s^{\prime}, \mathbf{r}_{-u}\right)$ for any $s^{\prime}<k$.

Therefore, when the property in Eq. (G.7) holds, the perturbed strategy profile $\mathbf{r}$ moves to the navigable small world $\left(\mathbf{r}^{\prime} \equiv k\right)$ in one synchronous step, as every player $u$ moves from its current strategy to its best response $r_{u}^{\prime}=k$. Also, it is clear that the property Eq. (G.7) consistently holds as any player takes one asynchronous step. This is because the asynchronous move only increases the number of nodes choosing a non-zero strategy, so the best response of every player is always $k$ after every asynchronous step. Thus, the perturbed strategy profile moves to the navigable small world as soon as every node takes at least one asynchronous step. Notice that the property in Eq. (G.7) holds with a probability of $1-1 / n$, so the theorem is proved. 


\section{H. PROOF OF THEOREM 4.1}

THEOREM 4.1. In the $k$-dimensional homogeneous DRB game, the optimal social welfare is $\Theta\left(\frac{n^{\alpha+k}}{\ln ^{\alpha+1} n}\right)$ for sufficiently large $n$.

Proof. Given the strategy profile $\mathbf{r}$, we partition the nodes $V$ into three sets: $V_{<k}=$ $\left\{v \in V \mid r_{v}<k\right\}, V_{>k}=\left\{v \in V \mid r_{v}>k\right\}, V_{=k}=\left\{v \in V \mid r_{v}=k\right\}$. So we have:

$$
\pi_{u}(\mathbf{r})=D\left(r_{u}\right)^{\alpha}\left(P_{u, V_{<k}}(\mathbf{r})+P_{u, V_{>k}}(\mathbf{r})+P_{u, V_{=k}}(\mathbf{r})\right) .
$$

For any node $u \in V_{=k}$, we have:

$$
P_{u, V_{<k}}(\mathbf{r})=\sum_{v \in V_{<k}} \frac{d_{M}(u, v)^{-r_{u}-r_{v}}}{c\left(r_{u}\right) c\left(r_{v}\right)} \leq \sum_{v \in V_{<k}} \frac{d_{M}(u, v)^{-r_{u}}}{c\left(r_{u}\right) c(k-\gamma)} \leq \frac{\sum_{\forall v \neq u} d_{M}(u, v)^{-r_{u}}}{c\left(r_{u}\right) c(k-\gamma)}=\frac{1}{c(k-\gamma)} .
$$

Combining the above inequality with bounds (A.2b), (A.1b) and (A.1a) on $D(k), c(k)$ and $c(k-\gamma)$, we get the upper bound on the payoff obtained from the set $V_{<k}$ :

$$
D\left(r_{u}=k\right)^{\alpha} P_{u, V_{<k}}(\mathbf{r}) \leq\left(\frac{2 \xi_{k}^{+} n}{\xi_{k}^{-} \ln n}\right)^{\alpha} \cdot \frac{2^{k+1} k}{\xi_{k}^{-} n^{\gamma}} \leq \frac{\left(\xi_{k}^{+}\right)^{\alpha} 2^{k+1+\alpha} k}{\left(\xi_{k}^{-}\right)^{\alpha+1}} \frac{n^{\alpha-\gamma}}{\ln ^{\alpha} n} .
$$

For the set $V_{>k}$, we have:

$$
\begin{aligned}
P_{u, V_{>k}}(\mathbf{r}) & =\sum_{v \in V_{>k}} \frac{d_{M}(u, v)^{-r_{u}-r_{v}}}{c\left(r_{u}\right) c\left(r_{v}\right)} \leq \frac{\sum_{j=1}^{n_{D}} b_{u}(j) \cdot j^{-r_{u}} \cdot j^{-k-\gamma}}{\xi_{k}^{-} c\left(r_{u}\right)}=\frac{\xi_{k}^{+} \sum_{j=1}^{n_{D}} j^{-1-r_{u}-\gamma}}{\xi_{k}^{-} c\left(r_{u}\right)} \\
& \leq \frac{\xi_{k}^{+}\left(1+\int_{1}^{n_{D}} x^{-1-r_{u}-\gamma} d x\right)}{\xi_{k}^{-} c\left(r_{u}\right)} \leq \frac{\xi_{k}^{+}\left(1+r_{u}+\gamma\right)}{\xi_{k}^{-}\left(r_{u}+\gamma\right) c\left(r_{u}\right)} \leq \frac{\xi_{k}^{+}(k+1)}{\xi_{k}^{-} \gamma c\left(r_{u}\right)} .
\end{aligned}
$$

Combining the above inequality with the bounds (A.2b), (A.1b) on $D(k), c(k)$, we get the upper bound on the payoff obtained from the set $V_{>k}$ :

$$
D\left(r_{u}=k\right)^{\alpha} P_{u, V_{>k}}(\mathbf{r}) \leq\left(\frac{2 \xi_{k}^{+} n}{\xi_{k}^{-} \ln n}\right)^{\alpha} \cdot \frac{2 \xi_{k}^{+}(k+1)}{\left(\xi_{k}^{-}\right)^{2} \gamma \ln n} \leq \frac{2^{\alpha+2}\left(\xi_{k}^{+}\right)^{\alpha+1} k}{\gamma\left(\xi_{k}^{-}\right)^{\alpha+2}} \frac{n^{\alpha}}{\ln ^{\alpha+1} n} .
$$

For the set $V_{=k}$, by Lemma A.4 we know that:

$$
D\left(r_{u}=k\right) P_{u, V_{=k} \backslash\{u\}}(\mathbf{r}) \leq \pi\left(r_{u}=k, \mathbf{r}_{-u} \equiv k\right) \leq \frac{2^{\alpha+3}\left(\xi_{k}^{+}\right)^{\alpha+1}}{\left(\xi_{k}^{-}\right)^{2+\alpha}} \cdot \frac{n^{\alpha}}{\ln ^{\alpha+2} n}
$$

Then, for any node $u \in V_{=k}$ and sufficiently large $n$, we have

$$
\begin{aligned}
& \pi_{u}(\mathbf{r})=D\left(r_{u}\right)^{\alpha}\left(P_{u, V_{<k}}(\mathbf{r})+P_{u, V_{>k}}(\mathbf{r})+P_{u, V_{=k} \backslash\{u\}}(\mathbf{r})\right) \\
& \leq \frac{\left(\xi_{k}^{+}\right)^{\alpha} 2^{k+1+\alpha} k}{\left(\xi_{k}^{-}\right)^{\alpha+1}} \frac{n^{\alpha-\gamma}}{\ln ^{\alpha} n}+\frac{2^{\alpha+2}\left(\xi_{k}^{+}\right)^{\alpha+1} k}{\gamma\left(\xi_{k}^{-}\right)^{\alpha+2}} \frac{n^{\alpha}}{\ln ^{\alpha+1} n}+\frac{2^{\alpha+3}\left(\xi_{k}^{+}\right)^{\alpha+1}}{\left(\xi_{k}^{-}\right)^{2+\alpha}} \cdot \frac{n^{\alpha}}{\ln ^{\alpha+2} n} \\
& <\frac{2^{\alpha+3}\left(\xi_{k}^{+}\right)^{\alpha+1} k}{\gamma\left(\xi_{k}^{-}\right)^{\alpha+2}} \frac{n^{\alpha}}{\ln ^{\alpha+1} n} .
\end{aligned}
$$

By Lemma A.3, for any node $u \notin V_{=k}$, there is a constant $\kappa$ such that for all sufficiently large $n, \pi\left(r_{u}, \mathbf{r}_{-u}\right) \leq \kappa n^{\alpha-\min \{1, \alpha\} \gamma}$.

So we have, for sufficiently large $n$, the social welfare of the profile is:

$$
\begin{aligned}
S W(\mathbf{r})= & \sum_{u \in V} \pi\left(r_{u}, \mathbf{r}_{-u}\right)<|V|\left(\frac{2^{\alpha+3}\left(\xi_{k}^{+}\right)^{\alpha+1} k}{\gamma\left(\xi_{k}^{-}\right)^{\alpha+2}} \frac{n^{\alpha}}{\ln ^{\alpha+1} n}+n^{\alpha-\min \{1, \alpha\} \gamma}\right) \\
& <\frac{2^{\alpha+4}\left(\xi_{k}^{+}\right)^{\alpha+1} k}{\gamma\left(\xi_{k}^{-}\right)^{\alpha+2}} \frac{n^{\alpha+k}}{\ln ^{\alpha+1} n} .
\end{aligned}
$$


The above inequality shows that the social welfare of the profile is at most $O\left(\frac{n^{\alpha+k}}{\ln ^{\alpha+1} n}\right)$.

Next, we construct the profile $\mathbf{r}$ as follows: for any node with location $(i, j)$, we set its strategy as $k$ if $j \bmod 2=0$, otherwise, we set its strategy as $k+\gamma$.

Notice that for any node $u$ with $r_{u}=k$, it has at least one neighbor $v$ with $r_{v}>k$. We get:

$$
P_{u, V}>p_{u}\left(v, r_{u}\right) p_{v}\left(u, r_{v}\right)>\frac{1}{c(k) c(k+\gamma)} .
$$

Combining with bounds in (A.2b), (A.1b) and (A.1c) on $D(k), c(k)$ and $c(k+\gamma)$, for any node $u$ with $r_{u}=k$,

$$
\pi_{u}(\mathbf{r})>D\left(r_{u}\right)^{\alpha} P_{u, V}(\mathbf{r})>\frac{\left(\xi_{k}^{-}\right)^{\alpha} \gamma}{2^{\alpha}\left(\xi_{k}^{+}\right)^{2+\alpha}(1+\gamma)} \frac{n^{\alpha}}{\ln ^{\alpha+1}(2 k n)}>\frac{\left(\xi_{k}^{-}\right)^{\alpha} \gamma}{2^{2 \alpha+1}\left(\xi_{k}^{+}\right)^{2+\alpha}(1+\gamma)} \frac{n^{\alpha}}{\ln ^{\alpha+1}(k n)} .
$$

So we have, for sufficiently large $n$, the social welfare of the profile is:

$S W(\mathbf{r})=\sum_{u \in V} \pi\left(r_{u}, \mathbf{r}_{-u}\right)>\frac{|V|}{2} \frac{\left(\xi_{k}^{-}\right)^{\alpha} \gamma}{2^{2 \alpha+1}\left(\xi_{k}^{+}\right)^{2+\alpha}(1+\gamma)} \frac{n^{\alpha}}{\ln ^{\alpha+1}(k n)}>\frac{\left(\xi_{k}^{-}\right)^{\alpha} \gamma}{2^{2 \alpha+2}\left(\xi_{k}^{+}\right)^{2+\alpha}(1+\gamma)} \frac{n^{\alpha+k}}{\ln ^{\alpha+1}(k n)}$.

The above inequality shows that the optimal social welfare is at least $\Omega\left(\frac{n^{\alpha+k}}{\ln ^{\alpha+1} n}\right)$. Combining the results of Eq.(H.6) and Eq.(H.9), the theorem is proved.

\section{PROOF OF THEOREM 4.2}

THEOREM 4.2. In the $k$-dimensional homogeneous DRB game, for sufficiently large $n$, the PoS is $\Theta(\ln n)$ and the PoA is $\Theta\left(\frac{n^{k}}{\ln ^{\alpha+1} n}\right)$.

Proof. According to Lemma A.4, the payoff of each player in navigable NE $\mathbf{r} \equiv k$ is $\pi\left(r_{u}=k, \mathbf{r}_{-u} \equiv k\right)=\Theta\left(\frac{n^{\alpha}}{\ln ^{\alpha+2} n}\right)$, so the social welfare of navigable NE is $\Theta\left(\frac{n^{\alpha+k}}{\ln ^{\alpha+2} n}\right)$. Combining with Theorem 4.1, the price of stability $(\mathrm{PoS})$ is $\Theta(\ln n)$.

For random small world $\mathbf{r}_{-u} \equiv 0$, we have:

$$
P_{u}\left(r_{u}, \mathbf{r}_{-u} \equiv 0\right)=\sum_{v \in V} \frac{d_{M}(u, v)^{-r_{u}}}{c\left(r_{u}\right) c(0)}=\frac{1}{c(0)}
$$

It is easy to get:

$$
c(0)=n^{k}-1 \geq n^{k} / 2 .
$$

Thus, combining the above inequality with the distance bound (A.2a), we have the payoff of node $u$ gets from $V_{=0}$ :

$$
\pi\left(r_{u}, \mathbf{r}_{-u} \equiv 0\right) \leq \frac{2^{(k+1) \alpha+1} k^{\alpha(2+k)}\left(\xi_{k}^{+}\right)^{\alpha}}{\left(\xi_{k}^{-}\right)^{\alpha}} n^{\alpha-k} .
$$

According to the above inequality, it is easy to get that the social welfare of $\mathbf{r} \equiv 0$ is at most $O\left(n^{\alpha}\right)$. We now examine its lower bound. To do so, we first get the lower bound on distance.

$$
D\left(r_{u}=0\right) \geq \frac{\sum_{j=1}^{n / 2} b_{u}(j) \cdot j}{c(0)} \geq \frac{\xi_{k}^{-} \int_{1}^{n / 2} x^{k} d x}{c(0)} \geq \frac{\xi_{k}^{-}(n / 2-1)^{1+k}}{(1+k) c(0)}>\frac{\xi_{k}^{-}(n / 4)^{1+k}}{(1+k) c(0)},
$$


Combining the above inequality with Eq.(I.2) and Eq. (I.1), so we can get

$$
\pi\left(r_{u}=k, \mathbf{r}_{-u} \equiv 0\right)>\frac{\left(\xi_{k}^{-}\right)^{\alpha}}{4^{\alpha(1+k)} 2^{\alpha-1} k} n^{\alpha-k} .
$$

Therefore, the social welfare of the random small-world network $(\mathbf{r} \equiv 0)$ is $\Theta\left(n^{\alpha}\right)$. Combining with Theorem 4.1, the price of anarchy (PoA) is $\Theta\left(\frac{n^{k}}{\ln ^{\alpha+1} n}\right)$. 